\title{
A Pyridazine-Bridged Sandwiched Cluster Incorporating Planar Hexanuclear Cobalt Ring and Bivacant Phosphotungstate
}

Ling-Yu Guo, ${ }^{\dagger}{ }^{\perp}$ Su-Yuan Zeng,,${ }^{\S}{ }^{\perp}$ ZvonkoJagličić, ${ }^{\dagger}$ Qi-Dong Hu, ${ }^{\dagger}$ Shi-Xuan Wang, ${ }^{\dagger}$ Zhi Wang, ${ }^{\dagger}$ Di Sun* ${ }^{\dagger}$

${ }^{\dagger}$ Key Lab of Colloid and Interface Chemistry, Ministry of Education, School of Chemistry and Chemical

Engineering, Shandong University, Jinan, 250100, P. R. China.

Email: dsun@sdu.edu.cn

${ }^{\ddagger}$ Faculty of Civil and Geodetic Engineering \& Institute of Mathematics, Physics and Mechanics, University of Ljubljana, Jamova 2, 1000 Ljubljana, Slovenia.

${ }^{\S}$ School of Chemistry and Chemical Engineering, Liaocheng University, Liaocheng, People’s Republic of China.

${ }^{\perp}$ These authors contributed equally.. 


\section{Materials and General Methods}

Commercially available solvents and metal salts were used without further purification. IR spectra were recorded on a PerkinElmer Spectrum Two in the frequency range of 4000-400 $\mathrm{cm}^{-1}$. Elemental analyses for $\mathrm{C}, \mathrm{H}$, and $\mathrm{N}$ were performed on a PerkinElmer $2400 \mathrm{CHN}$ elemental analyzer and, for P, W, and Co, were determined with a PLASMASPEC (I) ICP atomic emission spectrometer. Powder X-ray diffraction (PXRD) data were collected on a Philips X'Pert Pro MPD X-ray diffractometer with $\mathrm{Cu} \mathrm{K}_{\alpha}$ radiation equipped with an X'Celerator detector. Thermo gravimetric analyses (TGA) were performed on a SHIMADZU DTG-60A thermal analyzer from room temperature to $800^{\circ} \mathrm{C}$ under nitrogen atmosphere at a heating rate of $10^{\circ} \mathrm{C} / \mathrm{min}$. The diffuse-reflectance spectrum were performed on UV-Vis spectrophotometer (Evolution 220, ISA-220 accessory, Thermo Scientific) using a built-in $10 \mathrm{~mm}$ silicon photodiode with a $60 \mathrm{~mm}$ Spectralon sphere. The variable-temperature magnetic susceptibilities were collected on a Magnetic Property Measurement System (MPMS), SQUID-VSM (superconducting quantum interference device-vibrating sample magnetometer) (Quantum Design, USA). The circular dichroism (CD) spectra were recorded on a JASCO 810 Circular Dichroism Spectropolarimeter. The electrochemical measurements were carried out on a CHI660E electrochemical workstation $(\mathrm{CH}$ Instruments, Shanghai Chenghua Co.) with a conventional three-electrode cell consisting of a Pt plate as the counter electrode. The $\mathrm{Ag} / \mathrm{AgCl}$ (3 $\mathrm{M} \mathrm{KCl}$ filled) was used as the reference electrode. Electrical contact was established with copper rod through the back of the electrode. Prior to measurement, the electrolyte was thoroughly bubbled by purging it with nitrogen. 


\section{X-Ray Crystallography.}

The intensity data of $\mathbf{1}$ and $\mathbf{2}$ were collected on a Bruker APEX II CCD diffractometer with a graphite-monochromated Mo $\mathrm{K} \alpha$ radiation source $(\lambda=0.710703 \AA)$. The collected frames were integrated with the Bruker SAINT package with a narrow frame algorithm. ${ }^{1}$ An empirical absorption correction based on symmetry equivalent reflections was applied using the SADABS program. ${ }^{2}$ The structures were solved by direct methods, and all non-hydrogen atoms were refined anisotropically by least-squares on $F^{2}$ using the SHELXTL program. ${ }^{3}$ The hydrogen atoms attached to carbon were placed in idealized positions and refined using a riding model to the atom to which they were attached. The molecular graphics were produced with Diamond 3.2.4. CCDC 1486444-1486445 contain the supplementary crystallographic data for this paper. These data can be obtained free of charge via www.ccdc.cam.ac.uk/data_request/cif. Crystal data for both compounds are given in Table 1 and selected bond lengths and angles are shown in Table S1.

(1) Blessing, R. H. Acta Crystallogr., Sect. A 1995, 51, 33.

(2) Sheldrick, G. M. SADABS 2.05; University Göttingen: Gottingen, Germany, 1997.

(3) SHELXTL 6.10; Bruker Analytical Instrumentation: Madison, WI, 2000.

(4) Pennington, W. T. J. Appl. Crystallogr., 1999, 32, 1 
Table S1. Selected bond lengths and angles for 1.

\begin{tabular}{|c|c|c|c|}
\hline W1-O3 & $1.684(13)$ & W17-O56 & $1.913(12)$ \\
\hline W1-O4 & $1.916(12)$ & W17-O62 & $2.402(10)$ \\
\hline W1-O5 & $1.949(10)$ & W17-O64 & $1.955(12)$ \\
\hline W1-O7 & $1.833(13)$ & W18-O60 & $2.527(11)$ \\
\hline W1-O12 & $1.879(10)$ & W18-O67 & $1.854(11)$ \\
\hline $\mathrm{W} 1-\mathrm{O} 26$ & $2.504(11)$ & W18-O68 & $1.848(11)$ \\
\hline W2-O1 & $1.720(12)$ & W18-O69 & $1.923(11)$ \\
\hline W2-O4 & $1.908(12)$ & $\mathrm{W} 18-\mathrm{O} 70$ & $1.972(12)$ \\
\hline W2-O6 & $1.911(11)$ & W18-O74 & $1.714(11)$ \\
\hline W2-O10 & $1.935(12)$ & W19-O62 & $2.514(10)$ \\
\hline W2-O11 & $1.870(10)$ & W19-O63 & $1.894(11)$ \\
\hline $\mathrm{W} 2-\mathrm{O} 27$ & $2.476(10)$ & W19-O64 & $1.894(12)$ \\
\hline $\mathrm{W} 3-\mathrm{O} 2$ & $1.699(13)$ & W19-O69 & $1.910(11)$ \\
\hline W3-O5 & $1.898(10)$ & W19-O71 & $1.905(12)$ \\
\hline W3-O6 & $1.924(11)$ & W19-O73 & $1.715(12)$ \\
\hline W3-O8 & $1.879(11)$ & W20-O61 & $2.511(11)$ \\
\hline W3-O9 & $1.918(11)$ & W20-O65 & $1.948(11)$ \\
\hline W3-O25 & $2.485(11)$ & W20-066 & $1.903(13)$ \\
\hline W4-O7 & $2.044(11)$ & W20-O70 & $1.880(11)$ \\
\hline W4-O13 & $1.950(10)$ & W20-O71 & $1.903(11)$ \\
\hline W4-O14 & $1.735(13)$ & W20-O72 & $1.648(14)$ \\
\hline W4-O15 & $1.927(10)$ & $\mathrm{Co} 1-\mathrm{Co} 2$ & $3.283(3)$ \\
\hline $\mathrm{W} 4-\mathrm{O} 26$ & $2.428(12)$ & Co1-Co3 & $3.406(3)$ \\
\hline W4-O30 & $1.789(11)$ & Co1-O30 & $1.992(11)$ \\
\hline W5-O8 & $1.999(12)$ & Co1-O31 & $1.979(12)$ \\
\hline W5-O15 & $1.909(10)$ & Co1-O33W & $2.106(10)$ \\
\hline W5-O16 & $1.729(11)$ & Co1-O35 & $2.072(11)$ \\
\hline W5-O17 & $1.966(10)$ & Co1-O36 & $2.256(11)$ \\
\hline W5-O25 & $2.462(10)$ & Co1-N1 & $2.130(14)$ \\
\hline W5-O31 & $1.804(12)$ & $\mathrm{Co} 2-\mathrm{Co} 4$ & $3.422(3)$ \\
\hline W6-O9 & $1.956(11)$ & $\mathrm{Co} 2-\mathrm{O} 28$ & $2.019(11)$ \\
\hline W6-O17 & $1.905(10)$ & $\mathrm{Co} 2-\mathrm{O} 29$ & $2.010(11)$ \\
\hline W6-O18 & $1.966(10)$ & Co2-O33W & $2.110(11)$ \\
\hline W6-O19 & $1.673(12)$ & Co2-O36 & $2.247(11)$ \\
\hline W6-O25 & $2.385(11)$ & $\mathrm{Co} 2-\mathrm{O} 37$ & $2.082(11)$ \\
\hline W6-O32 & $1.837(11)$ & Co2-N7 & $2.137(14)$ \\
\hline W7-O10 & $1.921(12)$ & Co3-Co6 & $3.457(3)$ \\
\hline W7-O18 & $1.880(9)$ & Co3-O29W & $2.092(11)$ \\
\hline W7-O20 & $1.710(11)$ & Co3-O30W & $2.088(11)$ \\
\hline W7-O21 & $1.945(11)$ & Co3-O35 & $2.047(11)$ \\
\hline
\end{tabular}




\begin{tabular}{|c|c|c|c|}
\hline W7-O27 & $2.440(11)$ & Co3-O38 & $2.078(12)$ \\
\hline W7-O33 & $1.858(12)$ & $\mathrm{Co} 3-\mathrm{N} 2$ & $2.186(14)$ \\
\hline W8-O11 & $1.994(10)$ & $\mathrm{Co} 3-\mathrm{N} 3$ & $2.174(12)$ \\
\hline W8-O21 & $1.933(11)$ & Co4-Co5 & $3.418(3)$ \\
\hline W8-O22 & $1.753(10)$ & Co4-O31W & $2.108(10)$ \\
\hline W8-O23 & $1.926(11)$ & $\mathrm{Co} 4-\mathrm{O} 32 \mathrm{~W}$ & $2.150(12)$ \\
\hline W8-O27 & $2.407(10)$ & Co4-O37 & $2.074(11)$ \\
\hline W8-O28 & $1.779(12)$ & Co4-O40 & $2.059(11)$ \\
\hline W9-O12 & $2.039(12)$ & Co4-N5 & $2.186(14)$ \\
\hline W9-O13 & $1.933(12)$ & Co4-N8 & $2.099(16)$ \\
\hline W9-O23 & $1.925(11)$ & Co5-Co6 & $3.222(3)$ \\
\hline W9-O24 & $1.725(10)$ & Co5-O34W & $2.033(11)$ \\
\hline W9-O26 & $2.413(11)$ & Co5-O40 & $2.085(11)$ \\
\hline W9-O29 & $1.796(11)$ & Co5-O42 & $2.073(11)$ \\
\hline W10-O32 & $2.025(11)$ & Co5-O46 & $2.038(13)$ \\
\hline W10-O33 & $1.991(11)$ & Co5-O59 & $2.225(10)$ \\
\hline W10-O34 & $1.724(12)$ & Co5-N6 & $2.085(15)$ \\
\hline W10-O35 & $1.854(11)$ & Co6-O34W & $2.007(11)$ \\
\hline W10-O36 & $2.402(10)$ & Co6-O38 & $2.111(11)$ \\
\hline W10-O37 & $1.832(11)$ & Co6-O44 & $2.018(12)$ \\
\hline W11-O38 & $1.815(11)$ & Co6-O45 & $1.993(11)$ \\
\hline W11-O39 & $1.730(11)$ & Co6-O59 & $2.243(11)$ \\
\hline W11-O40 & $1.828(12)$ & Co6-N4 & $2.079(15)$ \\
\hline W11-O41 & $2.015(11)$ & $\mathrm{P} 1-\mathrm{O} 25$ & $1.566(11)$ \\
\hline W11-O43 & $1.966(11)$ & $\mathrm{P} 1-\mathrm{O} 26$ & $1.555(12)$ \\
\hline W11-O59 & $2.383(10)$ & $\mathrm{P} 1-\mathrm{O} 27$ & $1.554(12)$ \\
\hline W12-O43 & $1.873(11)$ & P1-O36 & $1.538(11)$ \\
\hline W12-O52 & $1.907(10)$ & P2-O59 & $1.569(11)$ \\
\hline W12-O53 & $1.715(12)$ & P2-O60 & $1.539(10)$ \\
\hline W12-O54 & $1.935(12)$ & P2-O61 & $1.550(11)$ \\
\hline W12-O61 & $2.374(10)$ & P2-O62 & $1.524(11)$ \\
\hline W12-O65 & $1.927(12)$ & Co7-Co8 & $3.503(3)$ \\
\hline W13-O44 & $1.806(11)$ & Co7-Co9 & $3.279(3)$ \\
\hline W13-O50 & $1.903(11)$ & Co7-O35W & $2.060(11)$ \\
\hline W13-O51 & $1.712(12)$ & Co7-O36W & $2.145(13)$ \\
\hline W13-O52 & $1.962(10)$ & Co7-O75 & $2.068(11)$ \\
\hline W13-O61 & $2.460(10)$ & Co7-N9 & $2.184(14)$ \\
\hline W13-066 & $1.984(13)$ & Co7-N14 & $2.166(12)$ \\
\hline W14-O45 & $1.778(12)$ & Co7-N16 & $2.153(13)$ \\
\hline W14-O48 & $1.940(12)$ & Co8-Co9 & $3.468(3)$ \\
\hline W14-O49 & $1.720(12)$ & Co8-O37W & $2.058(12)$ \\
\hline W14-O50 & $1.946(11)$ & Co8-O39W & $2.085(11)$ \\
\hline W14-O60 & $2.434(10)$ & Co8-O41W & $2.106(10)$ \\
\hline
\end{tabular}




\begin{tabular}{|c|c|c|c|}
\hline W14-O67 & $2.031(12)$ & Co8-O75 & $2.078(10)$ \\
\hline W15-O46 & $1.744(13)$ & Co8-N10 & $2.151(14)$ \\
\hline $\mathrm{W} 15-\mathrm{O} 47$ & $1.689(13)$ & Co8-N11 & $2.213(13)$ \\
\hline $\mathrm{W} 15-\mathrm{O} 48$ & $1.949(11)$ & Co9-O38W & $2.100(12)$ \\
\hline W15-O58 & $1.981(11)$ & Co9-O40W & $2.024(11)$ \\
\hline W15-O60 & $2.440(11)$ & Co9-O75 & $2.044(10)$ \\
\hline W15-O68 & $2.021(11)$ & Co9-N12 & $2.197(12)$ \\
\hline $\mathrm{W} 16-\mathrm{O} 42$ & $1.758(12)$ & Co9-N13 & $2.119(14)$ \\
\hline W16-O56 & $1.946(12)$ & Co9-N15 & $2.121(13)$ \\
\hline W16-O57 & $1.707(11)$ & $\mathrm{Na1-O42W}$ & $2.01(3)$ \\
\hline W16-O58 & $1.877(12)$ & Na1-O43W & $1.94(4)$ \\
\hline W16-O62 & $2.449(10)$ & Na1-O44W & $2.27(5)$ \\
\hline W16-O63 & $2.007(12)$ & Na1-O45W & $2.00(3)$ \\
\hline W17-O41 & $1.844(11)$ & Na1-O46W & $1.94(4)$ \\
\hline W17-O54 & $1.901(12)$ & Na1-O49W & $1.90(3)$ \\
\hline W17-O55 & $1.734(10)$ & & \\
\hline O30-Co1-O33W & $88.8(4)$ & O42-Co5-O59 & $93.2(4)$ \\
\hline O30-Co1-O35 & $168.7(4)$ & O42-Co5-N6 & $96.0(5)$ \\
\hline O30-Co1-O36 & $91.0(4)$ & O46-Co5-O40 & $168.7(5)$ \\
\hline O30-Co1-N1 & $98.3(5)$ & O46-Co5-O42 & $85.0(4)$ \\
\hline O31-Co1-O30 & $87.6(5)$ & O46-Co5-O59 & $92.2(4)$ \\
\hline O31-Co1-O33W & $175.6(4)$ & O46-Co5-N6 & $97.5(5)$ \\
\hline O31-Co1-O35 & $93.5(5)$ & N6-Co5-O59 & $167.1(5)$ \\
\hline O31-Co1-O36 & $95.6(4)$ & O34W-Co6-O38 & $92.1(4)$ \\
\hline O31-Co1-N1 & $97.3(5)$ & O34W-Co6-O44 & $172.2(5)$ \\
\hline O33W-Co1-O36 & $82.0(4)$ & O34W-Co6-O59 & $81.0(4)$ \\
\hline O33W-Co1-N1 & $85.7(5)$ & O34W-Co6-N4 & $86.5(5)$ \\
\hline O35-Co1-O33W & $89.5(4)$ & O38-Co6-O59 & $77.5(4)$ \\
\hline O35-Co1-O36 & $77.7(4)$ & O44-Co6-O38 & $89.7(4)$ \\
\hline O35-Co1-N1 & $92.7(5)$ & O44-Co6-O59 & $91.9(4)$ \\
\hline N1-Co1-O36 & $164.4(5)$ & O44-Co6-N4 & $101.1(5)$ \\
\hline $\mathrm{O} 28-\mathrm{Co} 2-\mathrm{O} 33 \mathrm{~W}$ & $173.4(4)$ & O45-Co6-O34W & $92.2(5)$ \\
\hline $\mathrm{O} 28-\mathrm{Co} 2-\mathrm{O} 36$ & $91.8(4)$ & O45-Co6-O38 & $169.0(5)$ \\
\hline $\mathrm{O} 28-\mathrm{Co} 2-\mathrm{O} 37$ & $89.9(4)$ & O45-Co6-O44 & $84.7(5)$ \\
\hline O28-Co2-N7 & $103.1(5)$ & O45-Co6-O59 & $93.2(4)$ \\
\hline $\mathrm{O} 29-\mathrm{Co} 2-\mathrm{O} 28$ & $88.7(5)$ & O45-Co6-N4 & $99.5(5)$ \\
\hline O29-Co2-O33W & $89.2(5)$ & N4-Co6-O38 & $90.9(5)$ \\
\hline $\mathrm{O} 29-\mathrm{Co} 2-\mathrm{O} 36$ & $93.2(4)$ & N4-Co6-O59 & $162.5(5)$ \\
\hline $\mathrm{O} 29-\mathrm{Co} 2-\mathrm{O} 37$ & $171.2(5)$ & O35W-Co7-O36W & $91.1(5)$ \\
\hline O29-Co2-N7 & $96.5(5)$ & O35W-Co7-O75 & $175.0(4)$ \\
\hline $\mathrm{O} 33 \mathrm{~W}-\mathrm{Co} 2-\mathrm{O} 36$ & $82.1(4)$ & O35W-Co7-N9 & $93.0(5)$ \\
\hline O33W-Co2-N7 & $83.3(5)$ & O35W-Co7-N14 & $90.2(5)$ \\
\hline
\end{tabular}




\begin{tabular}{|c|c|c|c|}
\hline O37-Co2-O33W & $91.2(4)$ & O35W-Co7-N16 & $91.1(5)$ \\
\hline $\mathrm{O} 37-\mathrm{Co} 2-\mathrm{O} 36$ & $78.2(4)$ & O36W-Co7-N9 & $85.0(5)$ \\
\hline O37-Co2-N7 & $92.3(5)$ & O36W-Co7-N14 & $177.4(5)$ \\
\hline N7-Co2-O36 & $162.4(4)$ & O36W-Co7-N16 & $87.2(5)$ \\
\hline O29W-Co3-N2 & $88.0(5)$ & O75-Co7-O36W & $92.7(5)$ \\
\hline O29W-Co3-N3 & $87.4(5)$ & O75-Co7-N9 & $90.5(4)$ \\
\hline O30W-Co3-O29W & $89.1(5)$ & O75-Co7-N14 & $86.1(4)$ \\
\hline O30W-Co3-N2 & $83.8(5)$ & O75-Co7-N16 & $85.9(5)$ \\
\hline O30W-Co3-N3 & $86.8(4)$ & N14-Co7-N9 & $92.7(5)$ \\
\hline $\mathrm{O} 35-\mathrm{Co} 3-\mathrm{O} 29 \mathrm{~W}$ & $177.6(5)$ & N16-Co7-N9 & $171.3(5)$ \\
\hline O35-Co3-O30W & $89.2(4)$ & N16-Co7-N14 & $95.0(5)$ \\
\hline O35-Co3-O38 & $91.9(4)$ & O37W-Co8-O39W & $86.7(4)$ \\
\hline $\mathrm{O} 35-\mathrm{Co} 3-\mathrm{N} 2$ & $90.1(5)$ & O37W-Co8-O41W & $171.9(4)$ \\
\hline $\mathrm{O} 35-\mathrm{Co} 3-\mathrm{N} 3$ & $94.2(5)$ & O37W-Co8-O75 & $88.0(4)$ \\
\hline O38-Co3-O29W & $89.9(4)$ & O37W-Co8-N10 & $91.6(5)$ \\
\hline O38-Co3-O30W & $176.6(4)$ & O37W-Co8-N11 & $98.8(5)$ \\
\hline $\mathrm{O} 38-\mathrm{Co} 3-\mathrm{N} 2$ & $99.5(5)$ & O39W-Co8-O41W & $86.0(4)$ \\
\hline $\mathrm{O} 38-\mathrm{Co} 3-\mathrm{N} 3$ & $89.9(4)$ & O39W-Co8-N10 & $92.8(5)$ \\
\hline N3-Co3-N2 & $169.6(5)$ & O39W-Co8-N11 & $90.5(5)$ \\
\hline $\mathrm{O} 31 \mathrm{~W}-\mathrm{Co} 4-\mathrm{O} 32 \mathrm{~W}$ & $88.6(4)$ & O41W-Co8-N10 & $85.1(4)$ \\
\hline O31W-Co4-N5 & $85.5(5)$ & O41W-Co8-N11 & $84.9(4)$ \\
\hline O32W-Co4-N5 & $83.2(5)$ & O75-Co8-O39W & $174.2(4)$ \\
\hline O37-Co4-O31W & $89.9(4)$ & $\mathrm{O} 75-\mathrm{Co} 8-\mathrm{O} 41 \mathrm{~W}$ & $99.4(4)$ \\
\hline O37-Co4-O32W & $176.5(4)$ & O75-Co8-N10 & $89.7(5)$ \\
\hline O37-Co4-N5 & $99.9(5)$ & O75-Co8-N11 & $87.9(5)$ \\
\hline O37-Co4-N8 & $90.7(5)$ & N10-Co8-N11 & $169.2(5)$ \\
\hline $\mathrm{O} 40-\mathrm{Co} 4-\mathrm{O} 31 \mathrm{~W}$ & $176.6(4)$ & O38W-Co9-N12 & $84.1(5)$ \\
\hline O40-Co4-O32W & $89.1(4)$ & O38W-Co9-N13 & $178.2(5)$ \\
\hline $\mathrm{O} 40-\mathrm{Co} 4-\mathrm{O} 37$ & $92.5(4)$ & O38W-Co9-N15 & $86.8(5)$ \\
\hline O40-Co4-N5 & $91.6(5)$ & O40W-Co9-O38W & $88.8(5)$ \\
\hline O40-Co4-N8 & $93.7(5)$ & O40W-Co9-O75 & $178.6(5)$ \\
\hline N8-Co4-O31W & $88.8(5)$ & O40W-Co9-N12 & $88.8(5)$ \\
\hline N8-Co4-O32W & $86.1(5)$ & O40W-Co9-N13 & $92.7(5)$ \\
\hline N8-Co4-N5 & $167.9(6)$ & O40W-Co9-N15 & $90.6(5)$ \\
\hline $\mathrm{O} 34 \mathrm{~W}-\mathrm{Co} 5-\mathrm{O} 40$ & $91.6(4)$ & O75-Co9-O38W & $91.4(5)$ \\
\hline $\mathrm{O} 34 \mathrm{~W}-\mathrm{Co} 5-\mathrm{O} 42$ & $172.5(4)$ & O75-Co9-N12 & $92.6(5)$ \\
\hline O34W-Co5-O46 & $90.5(4)$ & O75-Co9-N13 & $87.1(5)$ \\
\hline O34W-Co5-O59 & $80.9(4)$ & O75-Co9-N15 & $88.0(5)$ \\
\hline O34W-Co5-N6 & $90.5(5)$ & N13-Co9-N12 & $95.0(5)$ \\
\hline O40-Co5-O59 & $77.2(4)$ & N13-Co9-N15 & $94.1(5)$ \\
\hline O40-Co5-N6 & $93.6(5)$ & N15-Co9-N12 & $170.9(5)$ \\
\hline $\mathrm{O} 42-\mathrm{Co} 5-\mathrm{O} 40$ & $91.6(4)$ & & \\
\hline
\end{tabular}


Table S2. Summary of $\alpha_{1-}\left[\mathrm{PW}_{10} \mathrm{O}_{37}\right]^{9-}$ based inorganic-organic hybrids.

\begin{tabular}{|l|l|}
\hline $\mathrm{K}_{6} \mathrm{H}\left[\left[\left\{\mathrm{Ti}(\mathrm{ox})\left(\mathrm{H}_{2} \mathrm{O}\right)\right\}_{4}(\mu-\mathrm{O})_{3}\right]\left(\alpha-\mathrm{PW}_{10} \mathrm{O}_{37}\right)\right] \cdot 0.5 \mathrm{KCl} \cdot 10 \mathrm{H}_{2} \mathrm{O}$ & Inorg. Chem. 2006, 45, 8078-8085 \\
$\mathrm{K}_{6} \mathrm{H}\left[\left\{\mathrm{Ti}(\mathrm{ox})\left(\mathrm{H}_{2} \mathrm{O}\right)\right\}_{4}(\mu-\mathrm{O})_{3}\left(\mathrm{PW}_{10} \mathrm{O}_{37}\right)\right] \cdot 10 \mathrm{H}_{2} \mathrm{O}\left(\mathrm{H}_{2} \mathrm{Ox}=\right.$ oxalic acid $)$ & \\
\hline $\mathrm{Na}_{7} \mathrm{~K}_{5}\left[\left\{\mathrm{Co}(\mathrm{en})(\mu-\mathrm{OH})_{2} \mathrm{Co}(\mathrm{en})\right\}\left\{\mathrm{PW}_{10} \mathrm{O}_{37} \mathrm{Co}(\mathrm{en})\right\}_{2}\right] \cdot 20 \mathrm{H}_{2} \mathrm{O}(\mathrm{en}=$ ethanediamine $)$ & Chem. Commun., 2005, 5760-5762 \\
\hline$\left(\mathrm{Me}_{4} \mathrm{~N}\right)_{10}\left[\mathrm{Fe}_{4}(\mathrm{OH})_{4}\left(\mathrm{PW}_{10} \mathrm{O}_{37}\right)_{2}\right] \cdot 15 \mathrm{H}_{2} \mathrm{O}$ & Polyhedron 1998,17, 3721-3725 \\
\hline $\mathrm{KRb}_{5}\left[\left(\mathrm{PW}_{10} \mathrm{O}_{37}\right)\left(\mathrm{Ni}_{2}\left(\mathrm{H}_{2} \mathrm{O}\right)\right)_{2}\left(\mu-\mathrm{N}_{3}\right)\right] \cdot 19 \mathrm{H}_{2} \mathrm{O}$ & Angew. Chem. Int. Ed. 2004, 43, 2274-2277 \\
\hline $\mathrm{K}_{4} \mathrm{H}_{6}\left[\mathrm{Zr}_{4}(\mathrm{OH})_{6}\left(\mathrm{CH}_{3} \mathrm{COO}\right)_{2}\left(\mu-\mathrm{PW}_{10} \mathrm{O}_{37}\right)_{2}\right] \cdot 23 \mathrm{H}_{2} \mathrm{O}$ & Eur. J. Inorg. Chem. 2010, 3473-3477 \\
\hline $\mathrm{K}_{6}\left[\left(\mathrm{PW}{ }_{10} \mathrm{O}_{37}\right)\left(\mathrm{Ni}\left(\mathrm{H}_{2} \mathrm{O}\right)\right)_{2}\left(\mu-1,1-\mathrm{N}_{3}\right)\right]$ & Chem. Commun., 2006, 3477-3485 \\
\hline $\left.\mathrm{K}_{[}\left\{\left\{\mathrm{Ti}\left(\mathrm{H}_{2} \mathrm{O}\right)_{3}\right\}_{2}\left\{\mathrm{Ti}\left(\mathrm{H}_{2} \mathrm{O}\right)_{2}\right\}_{2}(\mu-\mathrm{O})_{3}\left(\mathrm{SO}_{4}\right)\right]\left(\mathrm{PW}_{10} \mathrm{O}_{37}\right)\right] \cdot \mathrm{nH} \mathrm{H}_{2} \mathrm{O}(\mathrm{n}=2-9)$ & Inorg. Chem. Commun2012, 19, 10-14 \\
\hline $\mathrm{Na}_{8}\left[\left\{\mathrm{M}_{4}\left(\mathrm{H}_{2} \mathrm{O}\right)_{4}(\mu-\mathrm{OH})_{2}\left(\mu \mu_{3}-\mathrm{O}\right)_{2}\right\}\left(\alpha-1,4-\mathrm{PW}_{10} \mathrm{O}_{37}\right)_{2}\right](\mathrm{M}=\mathrm{Hf}$ and M $=\mathrm{Zr})$ & Bull. Chem. Soc. Jpn. 2013, 86, 800-812 \\
\hline$\left(\mathrm{H}_{2} \mathrm{en}\right)\left[\mathrm{Cu}(\mathrm{en})_{2}\left(\mathrm{H}_{2} \mathrm{O}\right)\right]_{2}\left\{\left[\mathrm{Cu}{ }_{5}(\mathrm{en})_{2}(\mathrm{OH})_{2}\left(\mathrm{CH}_{3} \mathrm{COO}\right)_{2}\left(\mathrm{H}_{2} \mathrm{O}\right)_{8}\right]\left[\mathrm{Cu}{ }_{10.75}(\mathrm{en})_{6}(\mathrm{OH})_{4}\left(\mathrm{H}_{2} \mathrm{O}\right)_{5}(\alpha\right.\right.$ & Dalton Trans., 2015,44, 12546-12549 \\
$-\mathrm{PW}$ & \\
\hline
\end{tabular}

Table S3 Thecontrolled experiments and results by adjusting the $\mathrm{pH}$.

\begin{tabular}{|l|l|l|l|}
\hline $\mathrm{pH}$ & Experimental phenomena & $\mathrm{pH}$ & $\begin{array}{l}\text { Experimental } \\
\text { phenomena }\end{array}$ \\
\hline 1.47 & Yellow crystals of $\mathbf{2}$ & 5.14 & Red crystals of 1 \\
\hline 1.51 & Yellow crystals of $\mathbf{2}$ & 5.30 & Red crystals of $\mathbf{1}$ \\
\hline 1.60 & Yellow crystals of $\mathbf{2}$ & 5.50 & Red crystals of $\mathbf{1}$ \\
\hline 1.66 & Yellow crystals of $\mathbf{2}$ & 5.77 & Red precipitates \\
\hline 1.76 & Yellow crystals of $\mathbf{2}$ & 5.98 & Red precipitates \\
\hline 1.89 & Red precipitates & 6.16 & Red precipitates \\
\hline 2.02 & Red precipitates & 6.42 & Red precipitates \\
\hline 2.23 & Red precipitates & 6.69 & Pale blue precipitates \\
\hline 2.47 & Red precipitates & 7.05 & Pale blue precipitates \\
\hline 2.96 & Red precipitates & 7.43 & Pale blue precipitates \\
\hline 3.11 & Red precipitates & 7.72 & Pale blue precipitates \\
\hline 3.33 & Red precipitates & 7.94 & Pale blue precipitates \\
\hline 3.56 & Red precipitates & 8.12 & Pale blue precipitates \\
\hline 3.72 & Red precipitates & 8.38 & Pale blue precipitates \\
\hline 4.07 & Red crystals of $\mathbf{1}$ & 8.78 & Pale blue precipitates \\
\hline 4.33 & Red crystals of $\mathbf{1}$ & 9.70 & Pale blue precipitates \\
\hline 4.56 & Red crystals of $\mathbf{1}$ & 11.02 & Pale blue precipitates \\
\hline 4.82 & Red crystals of $\mathbf{1}$ & & \\
\hline
\end{tabular}


Table S4. Bond valence sum calculations onCo and $W$ centers in1.

\begin{tabular}{|l|l|l|l|}
\hline Atom & Valence & Atom & Valence \\
\hline W1 & 6.428 & W17 & 6.151 \\
\hline W2 & 6.112 & W18 & 6.226 \\
\hline W3 & 6.230 & W19 & 6.170 \\
\hline W4 & 5.953 & W20 & 6.430 \\
\hline W5 & 6.010 & Co1 & 2.304 \\
\hline W6 & 6.332 & Co2 & 2.214 \\
\hline W7 & 6.259 & Co3 & 2.242 \\
\hline W8 & 6.082 & Co4 & 2.255 \\
\hline W9 & 6.053 & Co5 & 2.277 \\
\hline W10 & 6.021 & Co6 & 2.395 \\
\hline W11 & 6.243 & Co7 & 2.286 \\
\hline W12 & 6.157 & Co8 & 2.225 \\
\hline W13 & 6.157 & Co9 & 2.470 \\
\hline W14 & 6.068 & & \\
\hline W15 & 6.255 & & \\
\hline W16 & 6.441 & & \\
\hline
\end{tabular}

Figure S1. The IR for 1.

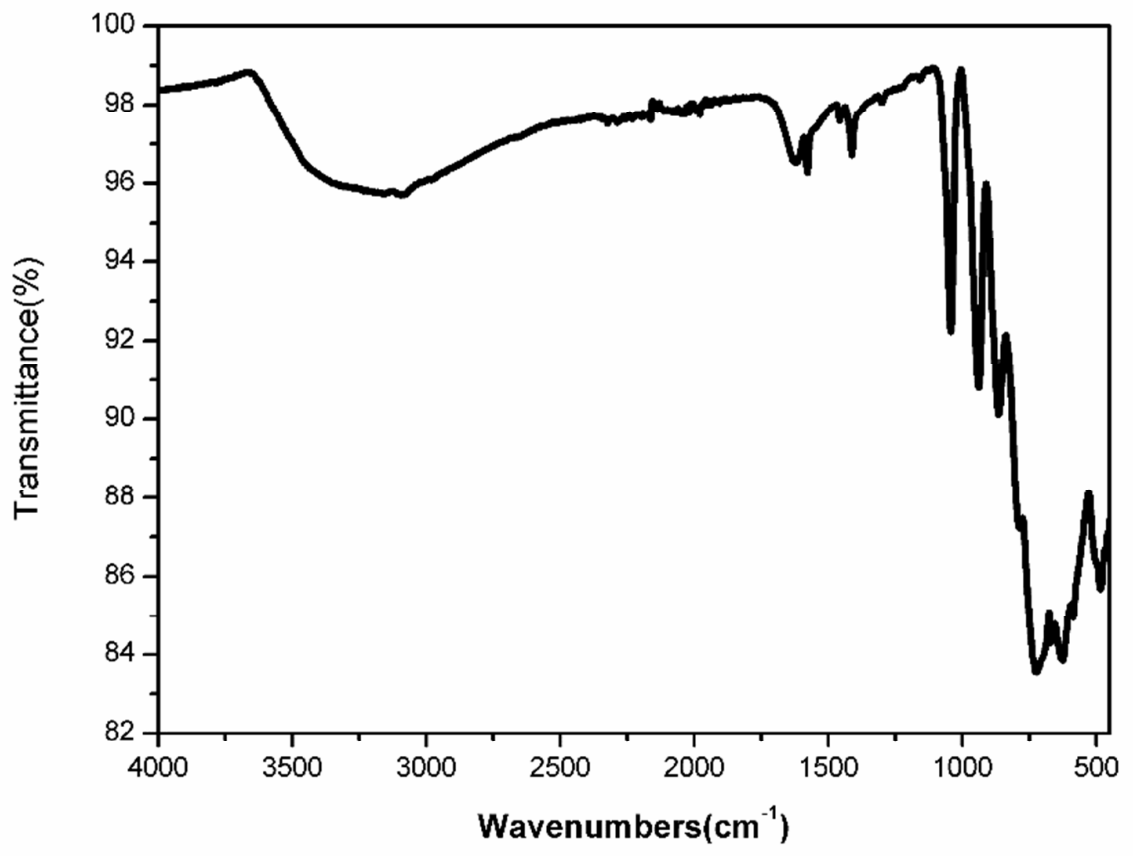


Figure S2. The solid state UV-Vis for 1 .

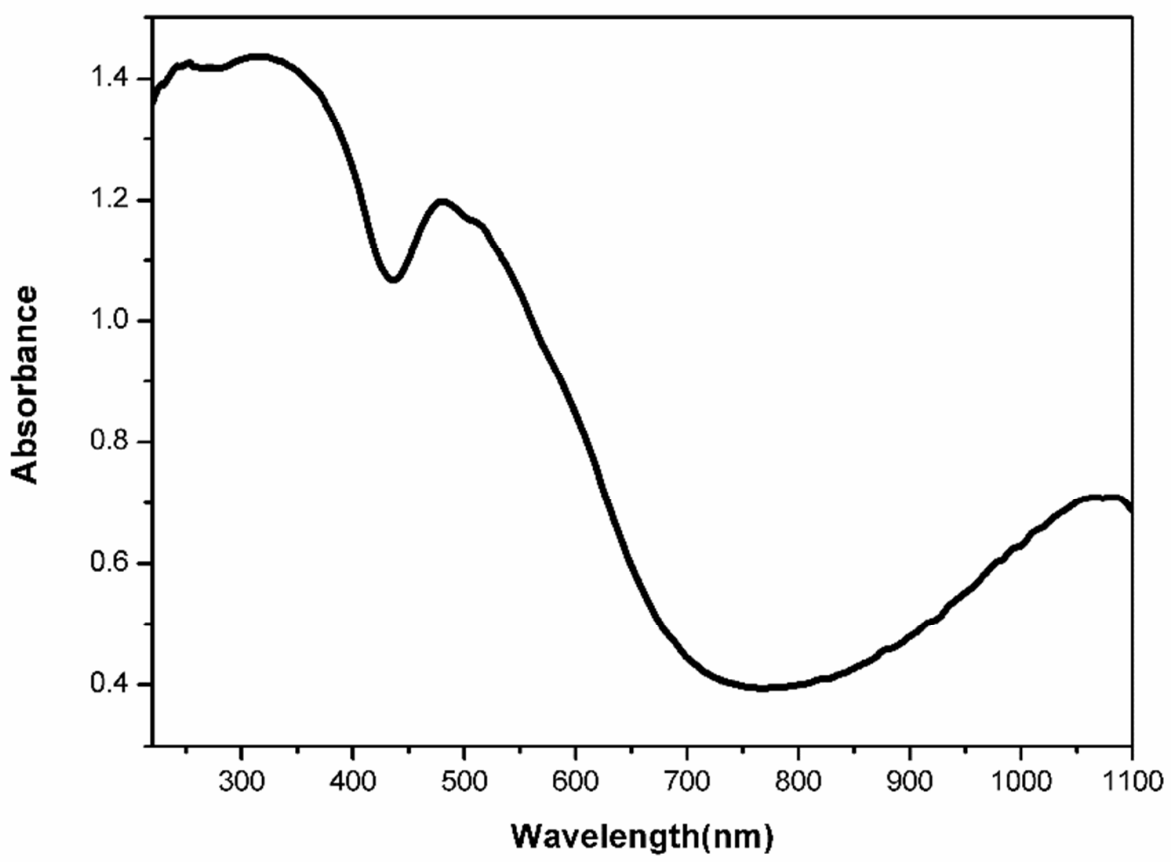

Figure S3. The compared PXRD patterns of 1.

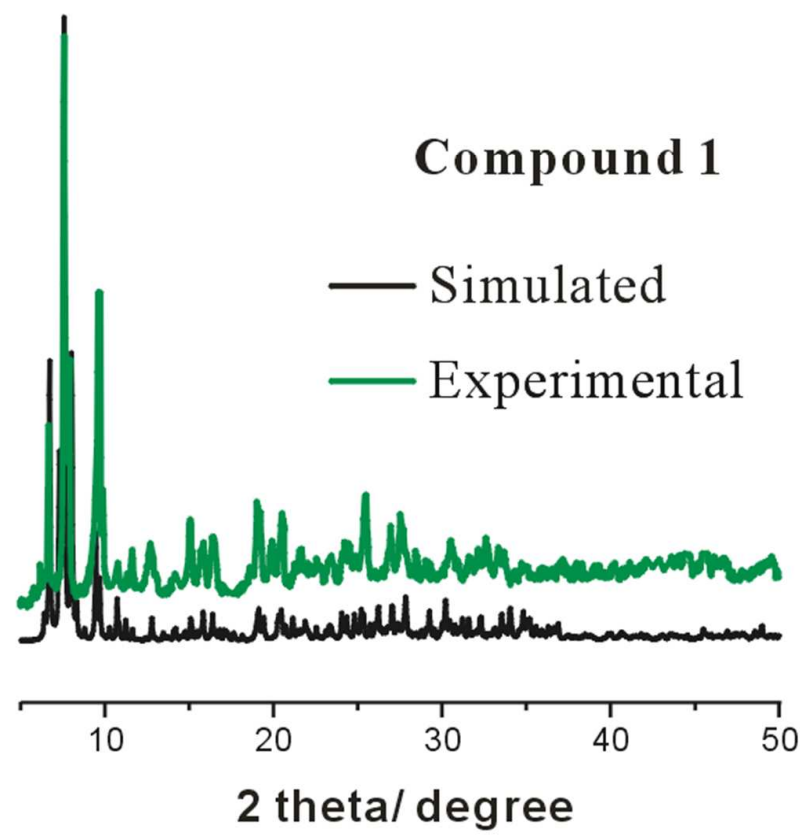


Figure S4. The TGA curve of 1.

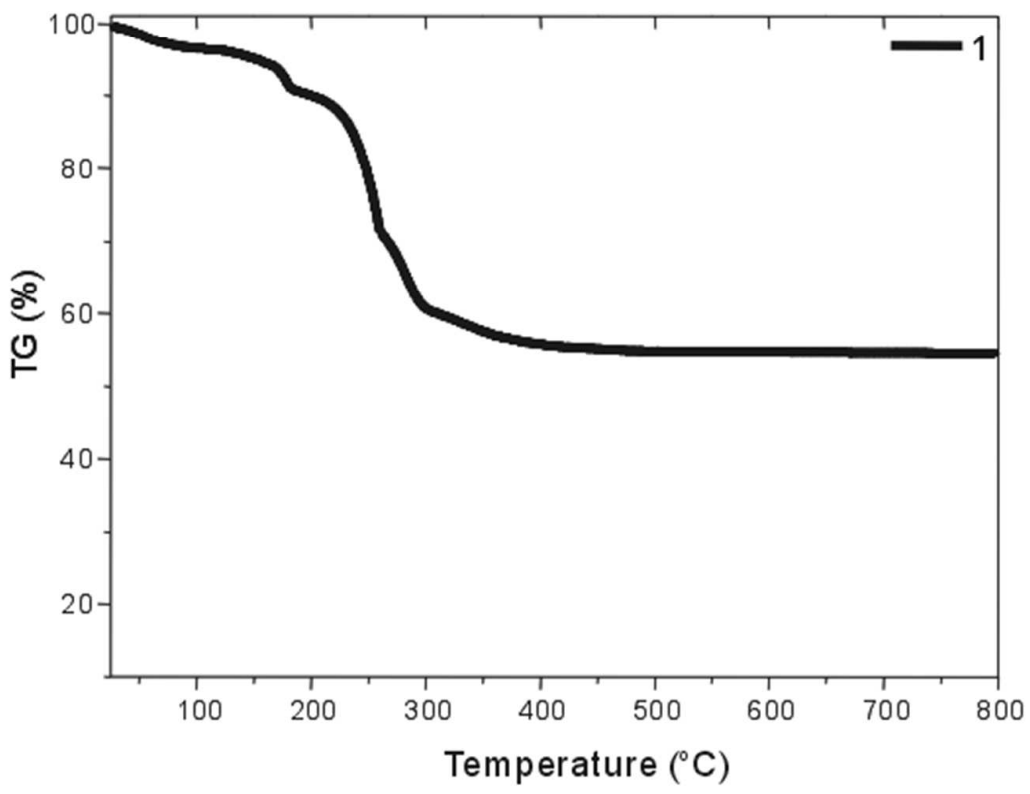


Figure S5. The CAT values toward the electrocatalytic reduction of nitrite.

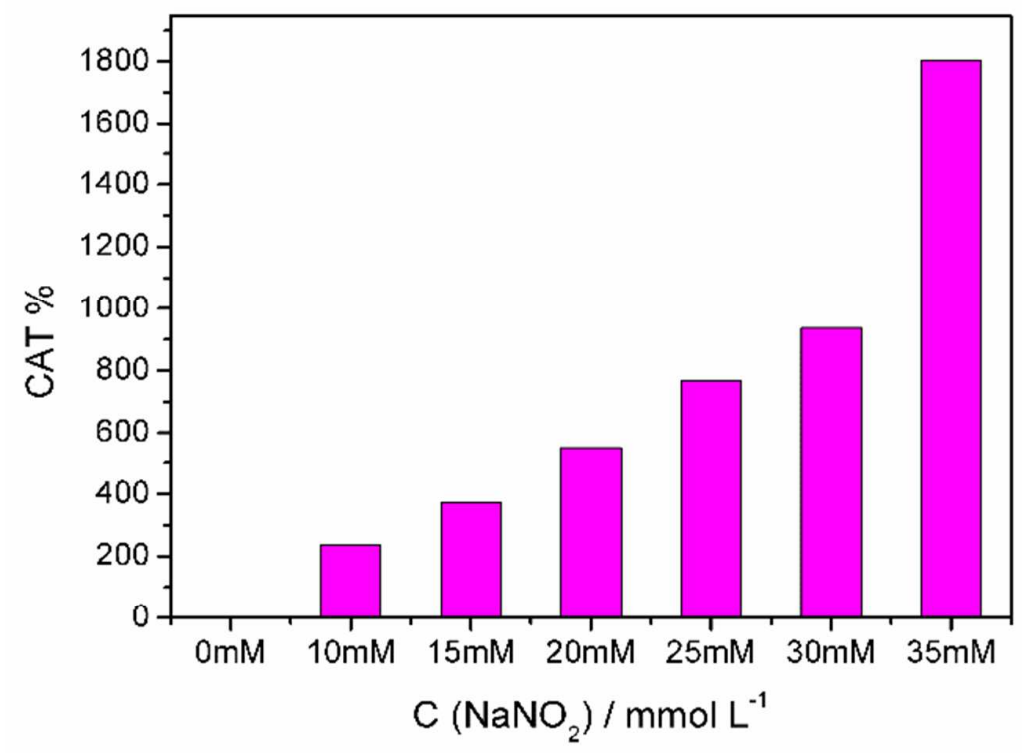

Figure S6. The CAT values toward the electrocatalytic reduction of hydrogen peroxide.

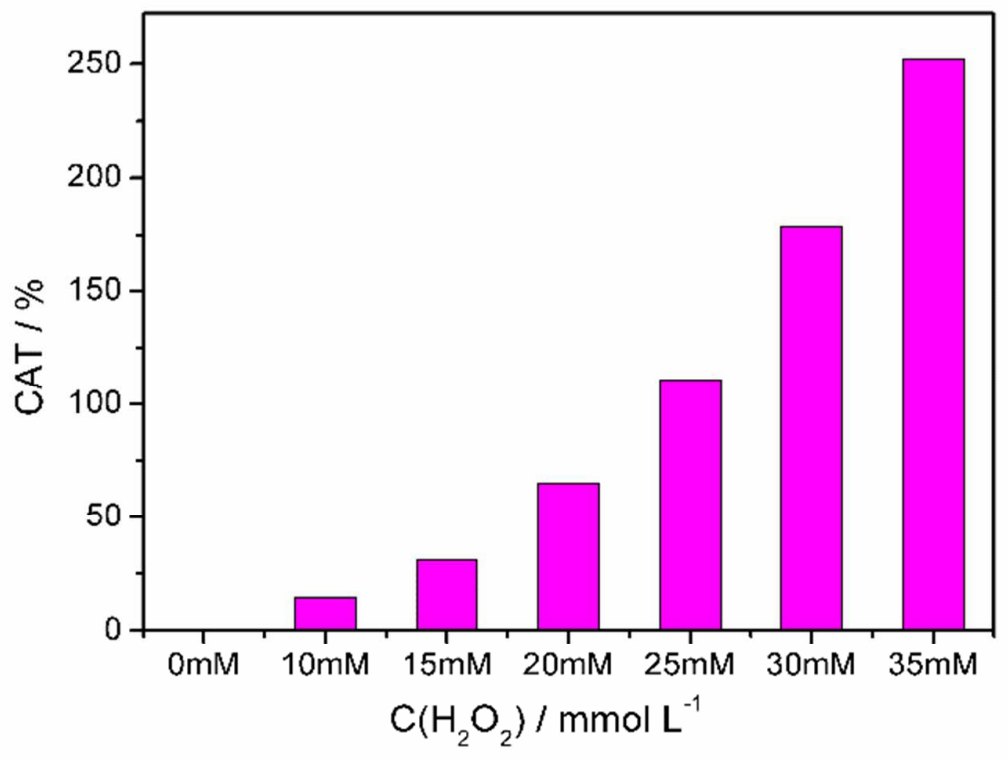


Figure S7. Susceptibility measured after zero field cooled (zfc) and field cooled (fc) protocols in three different magnetic fields. There is no difference between zfc and fe susceptibility and no field dependence of the susceptibility.

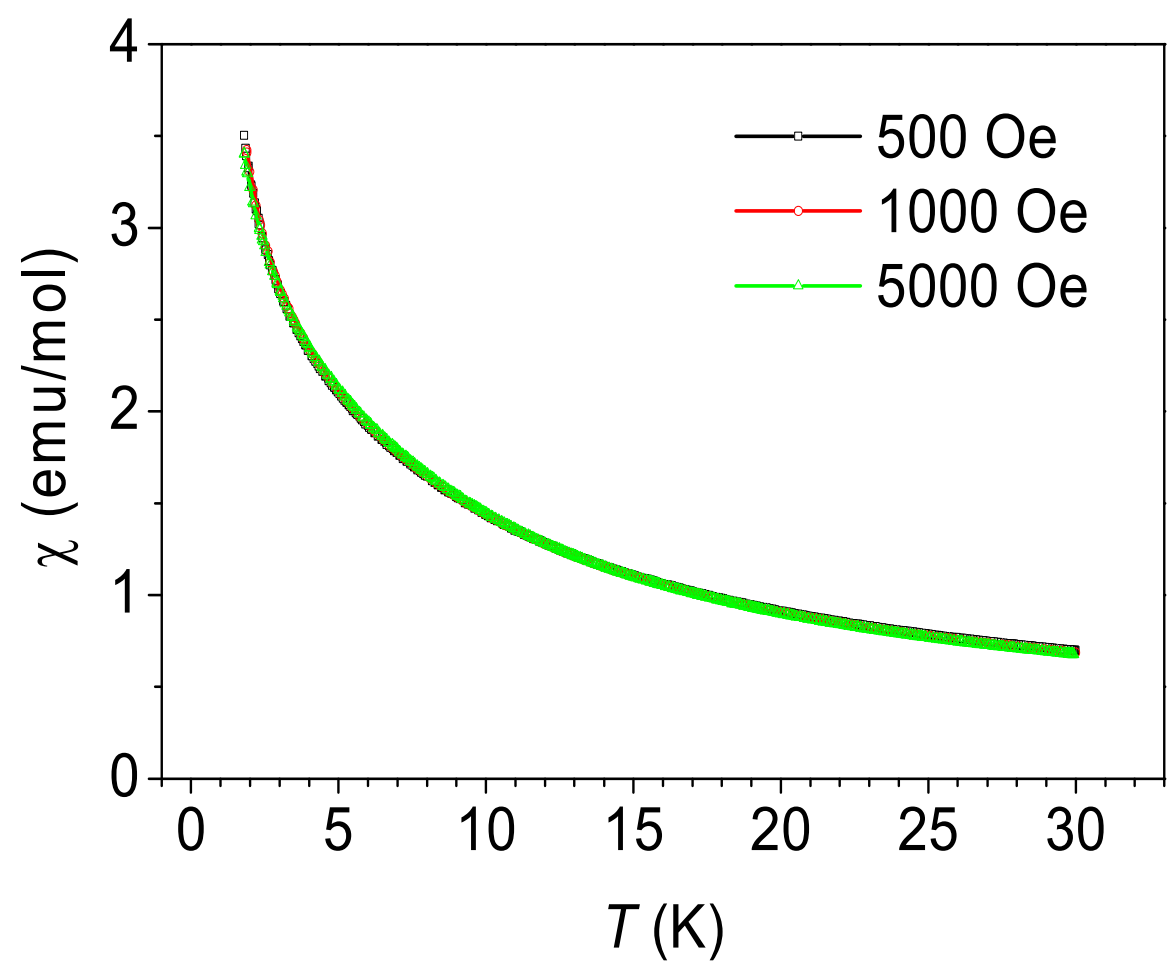


Figure S8. Ac susceptibility in low temperature range measured at several frequencies between $10 \mathrm{~Hz}$ and $1 \mathrm{kHz}$. There is no peak in $\chi^{\prime}(T)$ neither frequency dependence of the signal characteristic for magnetically frustrated system or single molecule magnetism.

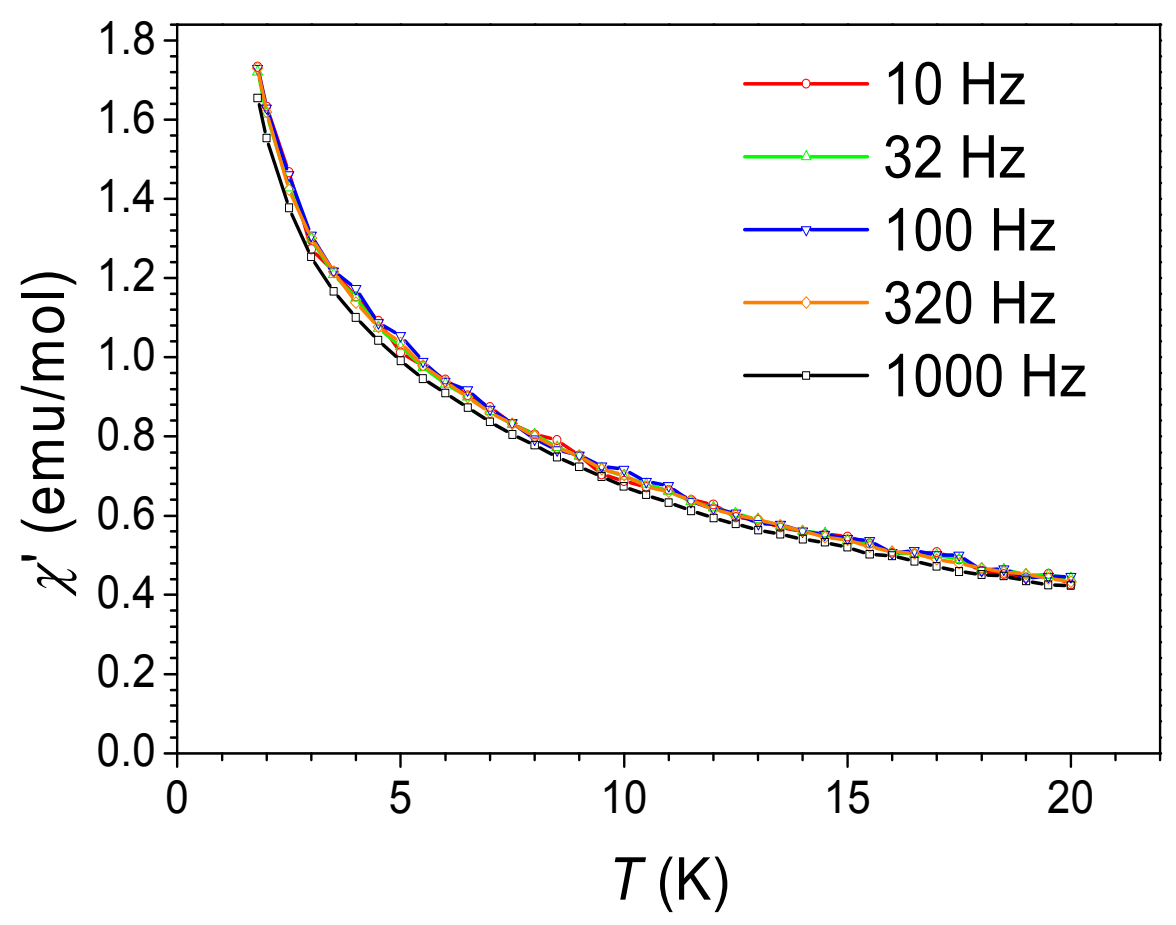


Figure S9. Polyhedral representation of molecular structure of 2.

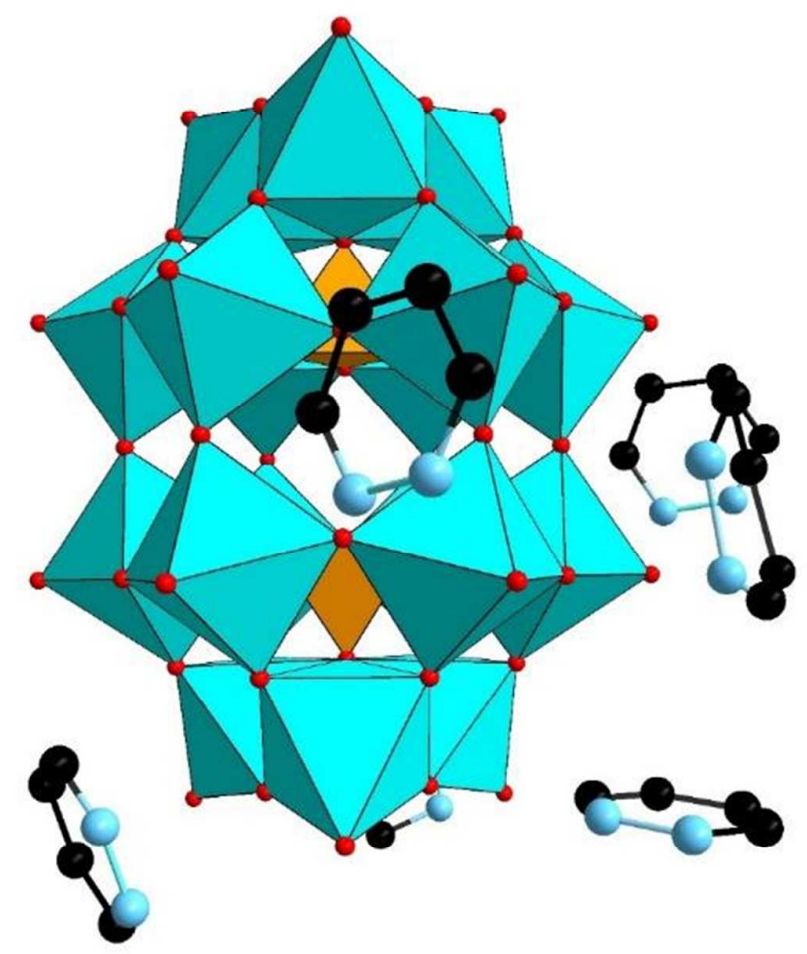

For polyhedron: $\mathrm{WO}_{6}$ : cyan; $\mathrm{PO}_{4}$ : brown.

For atom: W: cyan; $\mathrm{O}$, red; N, blue; $\mathrm{C}$, black. 
Table S5. Crystal data for 2.

\begin{tabular}{|c|c|}
\hline Empirical formula & $\mathrm{C}_{24} \mathrm{H}_{35} \mathrm{~N}_{12} \mathrm{O}_{66} \mathrm{P}_{2} \mathrm{~W}_{18}$ \\
\hline Formula weight & 4918.88 \\
\hline Temperature/K & 298.15 \\
\hline Crystal system & monoclinic \\
\hline Space group & $\mathrm{P} 2{ }_{1} / \mathrm{m}$ \\
\hline$a / \AA ̊$ & $14.0063(15)$ \\
\hline$b / \AA$ & $21.093(2)$ \\
\hline$c / \AA$ & $14.0094(15)$ \\
\hline$\alpha /^{\circ}$ & 90 \\
\hline$\beta /^{\circ}$ & $111.5448(9)$ \\
\hline$\gamma /{ }^{\circ}$ & 90 \\
\hline Volume $/ \AA^{3}$ & $3849.7(7)$ \\
\hline $\mathrm{Z}$ & 2 \\
\hline$\rho_{\text {calc }} \mathrm{g} / \mathrm{cm}^{3}$ & 4.243 \\
\hline$\mu / \mathrm{mm}^{-1}$ & 26.926 \\
\hline $\mathrm{F}(000)$ & 4306.0 \\
\hline Crystal size $/ \mathrm{mm}^{3}$ & $0.2 \times 0.15 \times 0.1$ \\
\hline Radiation & $\operatorname{MoK} \alpha(\lambda=0.71073)$ \\
\hline $2 \Theta$ range for data collection $/{ }^{\circ}$ & 4.964 to 49.99 \\
\hline Index ranges & $-16 \leq \mathrm{h} \leq 16,-25 \leq \mathrm{k} \leq 24,-16 \leq 1 \leq 16$ \\
\hline Reflections collected & 25310 \\
\hline Independent reflections & $6981\left[\mathrm{R}_{\mathrm{int}}=0.0585\right]$ \\
\hline Data/restraints/parameters & $6981 / 75 / 565$ \\
\hline Goodness-of-fit on $\mathrm{F}^{2}$ & 1.011 \\
\hline Final $\mathrm{R}$ indexes $[\mathrm{I}>=2 \sigma(\mathrm{I})]$ & $\mathrm{R}_{1}=0.0403, \mathrm{wR}_{2}=0.1057$ \\
\hline Final $\mathrm{R}$ indexes [all data] & $\mathrm{R}_{1}=0.0533, \mathrm{wR}_{2}=0.1158$ \\
\hline Largest diff. peak/hole / e $\AA^{-3}$ & $3.03 /-3.10$ \\
\hline
\end{tabular}


Table S6. Selected bond lengths and angles for 2.

\begin{tabular}{|c|c|c|c|}
\hline W1-O1 & $1.730(15)$ & W6-O29 ${ }^{\mathrm{i}}$ & $2.351(8)$ \\
\hline $\mathrm{W} 1-\mathrm{O} 2^{\mathrm{i}}$ & $1.910(9)$ & W6-O32 & $1.925(8)$ \\
\hline W1-O2 & $1.910(9)$ & W7-O19 & $1.872(8)$ \\
\hline W1-O7 & $1.909(9)$ & W7-O23 & $1.915(9)$ \\
\hline $\mathrm{W} 1-O 7^{\mathrm{i}}$ & $1.909(9)$ & W7-O24 & $1.704(10)$ \\
\hline W1-O8 & $2.366(13)$ & W7-O25 & $1.892(9)$ \\
\hline $\mathrm{W} 2-\mathrm{O} 2$ & $1.927(9)$ & $\mathrm{W}^{2}-\mathrm{O} 29^{\mathrm{i}}$ & $2.360(9)$ \\
\hline W2-O3 & $1.910(5)$ & W7-O31 & $1.954(8)$ \\
\hline $\mathrm{W} 2-\mathrm{O} 4$ & $1.715(9)$ & W8-O18 & $1.891(9)$ \\
\hline W2-O5 & $1.897(9)$ & W8-O25 & $1.910(9)$ \\
\hline W2-O6 & $1.908(8)$ & W8-O26 & $1.704(9)$ \\
\hline W2-O8 & $2.395(8)$ & W8-O27 & $1.908(6)$ \\
\hline W3-O5 & $1.931(9)$ & W8-O28 & $2.371(8)$ \\
\hline W3-O14 & $2.348(8)$ & W8-O30 & $1.930(8)$ \\
\hline W3-O16 & $1.707(9)$ & W9-O30 & $1.901(8)$ \\
\hline W3-O17 & $1.902(9)$ & W9-O31 & $1.869(9)$ \\
\hline W3-O18 & $1.895(9)$ & W9-O33 & $2.368(8)$ \\
\hline W3-O15 & $1.913(6)$ & W9-O35 & $1.923(8)$ \\
\hline W4-O6 & $1.900(9)$ & W9-O36 & $1.927(6)$ \\
\hline W4-O9 & $1.683(9)$ & W9-O37 & $1.727(9)$ \\
\hline W4-O10 & $1.903(10)$ & $\mathrm{W} 10-\mathrm{O} 32^{\mathrm{i}}$ & $1.891(9)$ \\
\hline W4-O13 & $2.372(9)$ & W10-O32 & $1.891(9)$ \\
\hline W4-O17 & $1.894(9)$ & W10-O33 & $2.397(11)$ \\
\hline W4-O19 & $1.928(8)$ & W10-O34 & $1.692(12)$ \\
\hline W5-O7 & $1.920(8)$ & W10-O35 & $1.925(9)$ \\
\hline W5-O10 & $1.922(9)$ & $\mathrm{W} 10-\mathrm{O} 35^{\mathrm{i}}$ & $1.925(9)$ \\
\hline W5-O11 & $1.688(10)$ & $\mathrm{P} 1-\mathrm{O} 8$ & $1.562(12)$ \\
\hline W5-O12 & $1.901(3)$ & $\mathrm{P} 1-\mathrm{O} 13^{\mathrm{i}}$ & $1.536(9)$ \\
\hline W5-O13 & $2.338(9)$ & P1-O13 & $1.536(9)$ \\
\hline W5-O20 & $1.885(8)$ & $\mathrm{P} 1-\mathrm{O} 14$ & $1.545(13)$ \\
\hline W6-O20 & $1.924(8)$ & $\mathrm{P} 2-\mathrm{O} 28$ & $1.525(11)$ \\
\hline W6-O21 & $1.894(3)$ & $\mathrm{P} 2-\mathrm{O} 29$ & $1.531(9)$ \\
\hline W6-O22 & $1.708(8)$ & $\mathrm{P} 2-\mathrm{O} 29^{\mathrm{i}}$ & $1.531(9)$ \\
\hline W6-O23 & $1.903(9)$ & $\mathrm{P} 2-\mathrm{O} 33$ & $1.584(12)$ \\
\hline
\end{tabular}

\begin{tabular}{|l|l|l|l|}
\hline $\mathrm{O} 1-\mathrm{W} 1-\mathrm{O} 2^{\mathrm{i}}$ & $101.0(4)$ & $\mathrm{O} 22-\mathrm{W} 6-\mathrm{O} 20$ & $98.4(4)$ \\
\hline $\mathrm{O} 1-\mathrm{W} 1-\mathrm{O} 2$ & $101.0(4)$ & $\mathrm{O} 22-\mathrm{W} 6-\mathrm{O} 21$ & $101.8(5)$ \\
\hline $\mathrm{O} 1-\mathrm{W} 1-\mathrm{O} 7$ & $101.7(4)$ & $\mathrm{O} 22-\mathrm{W} 6-\mathrm{O} 23$ & $100.2(4)$ \\
\hline $\mathrm{O}^{\mathrm{W}}-\mathrm{W} 1-\mathrm{O} 7^{\mathrm{i}}$ & $101.7(4)$ & $\mathrm{O} 22-\mathrm{W} 6-\mathrm{O} 29^{\mathrm{i}}$ & $173.4(4)$ \\
\hline
\end{tabular}




\begin{tabular}{|c|c|c|c|}
\hline O1-W1-O8 & $172.0(5)$ & O22-W6-O32 & $97.6(4)$ \\
\hline $\mathrm{O} 22^{\mathrm{i}}-\mathrm{W} 1-\mathrm{O} 2$ & $87.8(6)$ & O23-W6-O20 & $90.0(4)$ \\
\hline O2-W1-O8 & $73.3(3)$ & O23-W6-O29i & $73.2(3)$ \\
\hline $\mathrm{O} 2{ }^{\mathrm{i}}-\mathrm{W} 1-\mathrm{O} 8$ & $73.3(3)$ & O23-W6-O32 & $89.3(4)$ \\
\hline $\mathrm{O} 7^{\mathrm{i}}-\mathrm{W} 1-\mathrm{O} 2^{\mathrm{i}}$ & $89.3(4)$ & O32-W6-O29i & $81.6(3)$ \\
\hline $\mathrm{O} 7-\mathrm{W} 1-\mathrm{O} 2^{\mathrm{i}}$ & $157.2(4)$ & O19-W7-O23 & $92.1(4)$ \\
\hline $\mathrm{O} 7^{\mathrm{i}}-\mathrm{W} 1-\mathrm{O} 2$ & $157.2(4)$ & O19-W7-O25 & $89.5(4)$ \\
\hline O7-W1-O2 & $89.3(4)$ & O19-W7-O29i & $84.3(3)$ \\
\hline O7-W1-O7 ${ }^{\mathrm{i}}$ & $84.7(5)$ & O19-W7-O31 & $164.3(4)$ \\
\hline O7-W1-O8 & $84.2(3)$ & O23-W7-O29i & $72.8(3)$ \\
\hline O7'-W1-O8 & $84.2(3)$ & O23-W7-O31 & $88.2(4)$ \\
\hline O2-W2-O8 & $72.4(4)$ & O24-W7-O19 & $98.7(4)$ \\
\hline O3-W2-O2 & $88.2(5)$ & O24-W7-O23 & $99.5(4)$ \\
\hline O3-W2-O8 & $73.6(3)$ & $\mathrm{O} 24-\mathrm{W} 7-\mathrm{O} 25$ & $101.8(4)$ \\
\hline O4-W2-O2 & $100.8(4)$ & O24-W7-O29i & $171.9(4)$ \\
\hline O4-W2-O3 & $100.7(4)$ & O24-W7-O31 & $96.8(4)$ \\
\hline O4-W2-O5 & $102.7(4)$ & O25-W7-O23 & $158.1(4)$ \\
\hline O4-W2-O6 & $103.4(4)$ & $\mathrm{O} 25-\mathrm{W} 7-\mathrm{O} 29^{\mathrm{i}}$ & $85.7(3)$ \\
\hline O4-W2-O8 & $171.0(4)$ & O25-W7-O31 & $84.6(3)$ \\
\hline O5-W2-O2 & $156.5(4)$ & O31-W7-O29i & $80.8(3)$ \\
\hline O5-W2-O3 & $89.4(5)$ & O18-W8-O25 & $88.2(4)$ \\
\hline O5-W2-O6 & $85.2(4)$ & O18-W8-O27 & $91.0(4)$ \\
\hline O5-W2-O8 & $84.5(4)$ & O18-W8-O28 & $82.0(4)$ \\
\hline O6-W2-O2 & $87.5(4)$ & O18-W8-O30 & $162.5(4)$ \\
\hline O6-W2-O3 & $155.9(4)$ & $\mathrm{O} 25-\mathrm{W} 8-\mathrm{O} 28$ & $84.6(3)$ \\
\hline O6-W2-O8 & $82.5(3)$ & O25-W8-O30 & $85.1(4)$ \\
\hline O5-W3-O14 & $82.5(4)$ & O26-W8-O18 & $99.5(4)$ \\
\hline O16-W3-O5 & $98.6(4)$ & O26-W8-O25 & $101.0(4)$ \\
\hline O16-W3-O14 & $172.1(4)$ & O26-W8-O27 & $100.6(4)$ \\
\hline O16-W3-O17 & $102.9(4)$ & O26-W8-O28 & $174.2(4)$ \\
\hline O16-W3-O18 & $97.4(4)$ & O26-W8-O30 & $97.6(4)$ \\
\hline O16-W3-O15 & $99.0(5)$ & $\mathrm{O} 27-\mathrm{W} 8-\mathrm{O} 25$ & $158.2(4)$ \\
\hline O17-W3-O5 & $85.8(4)$ & O27-W8-O28 & $73.7(4)$ \\
\hline O17-W3-O14 & $85.0(3)$ & $\mathrm{O} 27-\mathrm{W} 8-\mathrm{O} 30$ & $89.3(4)$ \\
\hline O17-W3-O15 & $158.0(4)$ & O30-W8-O28 & $81.3(4)$ \\
\hline O18-W3-O5 & $163.8(4)$ & O30-W9-O33 & $83.6(4)$ \\
\hline O18-W3-O14 & $82.1(4)$ & O30-W9-O35 & $157.3(4)$ \\
\hline O18-W3-O17 & $88.0(4)$ & O30-W9-O36 & $86.8(4)$ \\
\hline O18-W3-O15 & $91.1(4)$ & O31-W9-O30 & $85.7(4)$ \\
\hline O15-W3-O5 & $89.1(4)$ & O31-W9-O33 & $84.4(3)$ \\
\hline O15-W3-O14 & $73.1(4)$ & O31-W9-O35 & $90.9(4)$ \\
\hline O6-W4-O10 & $91.2(4)$ & O31-W9-O36 & $155.6(4)$ \\
\hline O6-W4-O13 & $81.5(3)$ & O35-W9-O33 & $73.7(4)$ \\
\hline
\end{tabular}




\begin{tabular}{|c|c|c|c|}
\hline O6-W4-O19 & $163.5(4)$ & O35-W9-O36 & $87.2(4)$ \\
\hline O9-W4-O6 & $98.3(4)$ & O36-W9-O33 & $71.8(4)$ \\
\hline O9-W4-O10 & $100.5(4)$ & O37-W9-O30 & $102.1(4)$ \\
\hline O9-W4-O13 & $173.6(4)$ & O37-W9-O31 & $103.4(4)$ \\
\hline O9-W4-O17 & $101.3(4)$ & O37-W9-O33 & $170.5(4)$ \\
\hline O9-W4-019 & $98.0(4)$ & O37-W9-O35 & $100.5(4)$ \\
\hline O10-W4-O13 & $73.1(3)$ & O37-W9-O36 & $100.8(5)$ \\
\hline O10-W4-O19 & $88.5(4)$ & O32 ${ }^{\mathrm{i}}-\mathrm{W} 10-\mathrm{O} 32$ & $85.8(5)$ \\
\hline O17-W4-O6 & $86.9(4)$ & O32-W10-O33 & $83.5(3)$ \\
\hline O17-W4-O10 & $158.1(4)$ & O32 $-\mathrm{i} 10-\mathrm{O} 33$ & $83.5(3)$ \\
\hline O17-W4-O13 & $85.0(3)$ & O32 $-\mathrm{W} 10-\mathrm{O} 35$ & $156.4(3)$ \\
\hline O17-W4-O19 & $87.2(4)$ & O32-W10-O35 & $89.3(4)$ \\
\hline O19-W4-O13 & $82.6(3)$ & $\mathrm{O} 32^{\mathrm{i}}-\mathrm{W} 10-\mathrm{O} 35^{\mathrm{i}}$ & $89.3(4)$ \\
\hline O7-W5-O10 & $90.5(4)$ & O32-W10-O35 ${ }^{\mathrm{i}}$ & $156.4(3)$ \\
\hline O7-W5-O13 & $81.7(3)$ & O34-W10-O32 ${ }^{\mathrm{i}}$ & $104.1(4)$ \\
\hline O10-W5-O13 & $73.6(3)$ & O34-W10-O32 & $104.1(4)$ \\
\hline O11-W5-O7 & $97.3(4)$ & O34-W10-O33 & $169.4(6)$ \\
\hline O11-W5-O10 & $98.7(5)$ & O34-W10-O35 & $99.5(4)$ \\
\hline O11-W5-O12 & $104.1(5)$ & O34-W10-O35 & $99.5(4)$ \\
\hline O11-W5-O13 & $172.2(4)$ & O35-W10-O33 & $73.0(3)$ \\
\hline O11-W5-O20 & $97.7(4)$ & O35 ${ }^{\mathrm{i}}-\mathrm{W} 10-\mathrm{O} 33$ & $73.0(3)$ \\
\hline O12-W5-O7 & $86.7(4)$ & O35-W10-O35 ${ }^{\mathrm{i}}$ & $86.0(5)$ \\
\hline O12-W5-O10 & $157.2(5)$ & O13-P1-O8 & $106.8(4)$ \\
\hline O12-W5-O13 & $83.6(4)$ & O13 $-\mathrm{P} 1-\mathrm{O} 8$ & $106.8(4)$ \\
\hline O20-W5-O7 & $164.8(4)$ & O13-P1-O13 ${ }^{i}$ & $110.6(7)$ \\
\hline O20-W5-O10 & $89.5(4)$ & O13-P1-O14 & $112.5(4)$ \\
\hline O20-W5-O12 & $87.5(4)$ & O13 ${ }^{\mathrm{i}}-\mathrm{P} 1-\mathrm{O} 14$ & $112.5(4)$ \\
\hline O20-W5-O13 & $83.8(3)$ & O14-P1-O8 & $107.2(7)$ \\
\hline O20-W6-O29i & $82.8(3)$ & O28-P2-O29 & $112.3(4)$ \\
\hline O20-W6-O32 & $163.9(3)$ & $\mathrm{O} 28-\mathrm{P} 2-\mathrm{O} 29^{\mathrm{i}}$ & $112.3(4)$ \\
\hline O21-W6-O20 & $89.0(4)$ & $\mathrm{O} 28-\mathrm{P} 2-\mathrm{O} 33$ & $106.8(7)$ \\
\hline $\mathrm{O} 21-\mathrm{W} 6-\mathrm{O} 23$ & $157.9(5)$ & O29-P2-O29 & $111.4(7)$ \\
\hline O21-W6-O29 & $84.7(4)$ & O29-P2-O33 & $106.8(4)$ \\
\hline O21-W6-O32 & $85.6(4)$ & O29i'-P2-O33 & $106.8(4)$ \\
\hline
\end{tabular}


Figure S10. Polyhedral representation of totally molecular structure of 3.

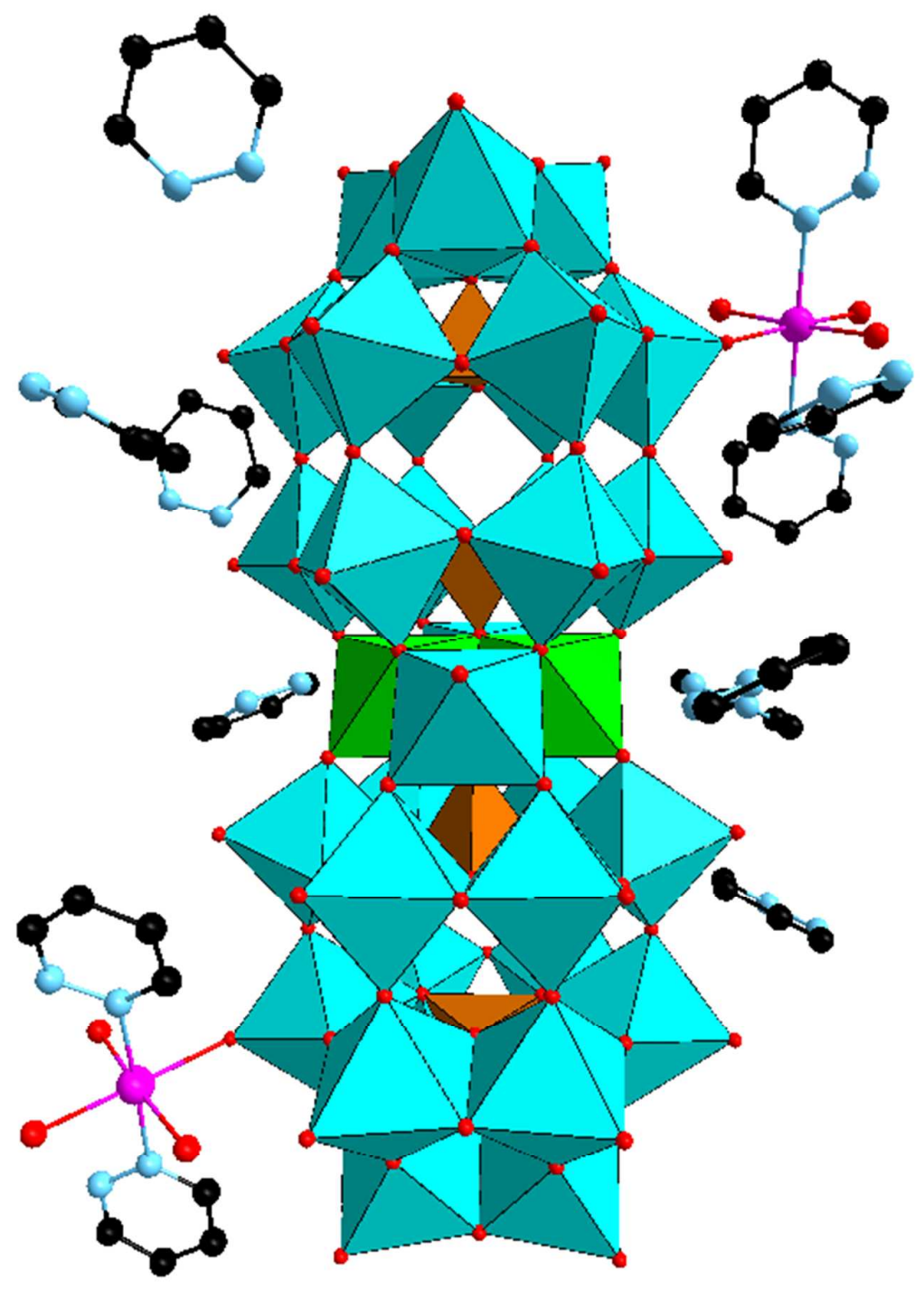

For polyhedron: $\mathrm{WO}_{6}$ : cyan; $\mathrm{NaO}_{6}$ : green; $\mathrm{PO}_{4}$ : brown.

For atom: W: cyan; Co: purple; O, red; N, blue; C, black. 
Table S7. Crystal data for 3.

\begin{tabular}{|l|l|}
\hline Empirical formula & $\mathrm{C}_{48} \mathrm{H}_{70} \mathrm{Co}_{2} \mathrm{~N}_{24} \mathrm{Na}_{2} \mathrm{O}_{130} \mathrm{P}_{4} \mathrm{~W}_{32}$ \\
\hline Formula weight & 9234.20 \\
\hline Temperature/K & 173.15 \\
\hline Crystal system & monoclinic \\
\hline Space group & $\mathrm{P}{ }_{1} / \mathrm{a}$ \\
\hline$a / \AA$ & $17.444(2)$ \\
\hline$b / \AA$ & $23.888(3)$ \\
\hline$c / \AA$ & $20.171(3)$ \\
\hline$\alpha /{ }^{\circ}$ & 90.00 \\
\hline$\beta /{ }^{\circ}$ & $94.2102(15)$ \\
\hline$\gamma /{ }^{\circ}$ & 90.00 \\
\hline Volume/ $\AA^{3}$ & $8383(2)$ \\
\hline $\mathrm{Z}$ & 2 \\
\hline$\rho_{\text {calcg} / \text { cm }^{3}}$ & 3.658 \\
\hline$\mu /$ mm $^{-1}$ & 22.198 \\
\hline $\mathrm{F}(000)$ & 8140.0 \\
\hline Crystal size/mm & $0.2 \times 0.15 \times 0.1$ \\
\hline Radiation & $\mathrm{MoK} \alpha(\lambda=0.71073)$ \\
\hline $2 \Theta$ range for data collection/ & 4.98 to 50 \\
\hline Index ranges & $-17 \leq \mathrm{h} \leq 20,-28 \leq \mathrm{k} \leq 27,-24 \leq 1 \leq 21$ \\
\hline Reflections collected & 40018 \\
\hline Independent reflections & $14740\left[\mathrm{R}_{\text {int }}=0.0711\right]$ \\
\hline Data/restraints/parameters & $14740 / 291 / 1084$ \\
\hline Goodness-of-fit on $\mathrm{F}^{2}$ & 0.987 \\
\hline Final R indexes [I $=2 \sigma(\mathrm{I})]$ & $\mathrm{R}_{1}=0.0556, \mathrm{wR}_{2}=0.1274$ \\
\hline Final R indexes [all data] & $\mathrm{R}_{1}=0.1167, \mathrm{wR}_{2}=0.1612$ \\
\hline Largest diff. peak/hole / e $\AA^{-3}$ & $2.47 /-1.80$ \\
\hline & \\
\hline & \\
\hline
\end{tabular}


Table S8. Selected bond lengths and angles for 3 .

\begin{tabular}{|c|c|c|c|}
\hline W1-O50 & $1.940(15)$ & W9-O26 & $2.381(15)$ \\
\hline $\mathrm{W} 1-\mathrm{O} 52^{\mathrm{i}}$ & $2.019(15)$ & W9-O33 & $1.841(15)$ \\
\hline $\mathrm{W} 1-\mathrm{O} 53^{\mathrm{i}}$ & $2.024(14)$ & W10-O12 & $2.036(16)$ \\
\hline W1-O55 & $1.955(15)$ & W10-O15 & $1.873(17)$ \\
\hline W1-O56 & $2.275(14)$ & W10-O16 & $1.736(16)$ \\
\hline W1-O57 & $1.848(19)$ & W10-O17 & $1.893(16)$ \\
\hline W1A-O51 & $1.995(14)$ & W10-O26 & $2.330(13)$ \\
\hline W1A-O52 & $2.141(15)$ & W10-O34 & $1.818(15)$ \\
\hline W1A-O53 ${ }^{\mathrm{i}}$ & $2.092(16)$ & W11-O11 & $1.960(18)$ \\
\hline W1A-O54 ${ }^{\mathrm{i}}$ & $1.998(13)$ & W11-O14 & $1.700(16)$ \\
\hline W1A-O56 ${ }^{\mathrm{i}}$ & $2.118(13)$ & W11-O15 & $1.911(16)$ \\
\hline W1A-O56 & $2.164(13)$ & $\mathrm{W} 11-\mathrm{O} 25$ & $1.936(15)$ \\
\hline W2-O32 & $1.954(14)$ & $\mathrm{W} 11-\mathrm{O} 27$ & $2.378(14)$ \\
\hline W2-O40 & $1.912(13)$ & W11-O29 & $1.887(14)$ \\
\hline W2-O41 & $1.714(15)$ & W12-O10 & $1.956(15)$ \\
\hline W2-O42 & $1.864(14)$ & $\mathrm{W} 12-\mathrm{O} 23$ & $1.917(15)$ \\
\hline W2-O48 & $2.343(14)$ & W12-O24 & $1.713(14)$ \\
\hline W2-O53 & $1.941(16)$ & $\mathrm{W} 12-\mathrm{O} 25$ & $1.890(16)$ \\
\hline W3-O33 & $1.976(15)$ & W12-O27 & $2.347(14)$ \\
\hline W3-O38 & $1.880(14)$ & W12-O30 & $1.863(14)$ \\
\hline W3-O39 & $1.739(16)$ & W13-O9 & $1.988(14)$ \\
\hline W3-O40 & $1.887(14)$ & W13-O21 & $1.913(17)$ \\
\hline W3-O49 & $2.351(14)$ & W13-O22 & $1.757(16)$ \\
\hline W3-O52 & $1.907(14)$ & $\mathrm{W} 13-\mathrm{O} 23$ & $1.856(15)$ \\
\hline W4-O34 & $2.025(15)$ & $\mathrm{W} 13-\mathrm{O} 28$ & $2.327(14)$ \\
\hline W4-O36 & $1.905(16)$ & W13-O31 & $1.830(14)$ \\
\hline W4-O37 & $1.727(16)$ & $\mathrm{W} 14-\mathrm{O} 2$ & $1.732(17)$ \\
\hline W4-O38 & $1.927(15)$ & W14-O4 & $1.945(14)$ \\
\hline W4-O49 & $2.316(13)$ & W14-O6 & $1.912(17)$ \\
\hline W4-O51 & $1.791(15)$ & W14-O7 & $1.874(17)$ \\
\hline W5-O29 & $1.930(14)$ & W14-O8 & $1.861(15)$ \\
\hline W5-O35 & $1.723(18)$ & W14-O13 & $2.370(14)$ \\
\hline W5-O36 & $1.892(16)$ & W15-O1 & $1.710(16)$ \\
\hline W5-O46 & $1.930(14)$ & W15-O5 & $1.914(16)$ \\
\hline W5-O47 & $2.352(13)$ & W15-O6 & $1.940(19)$ \\
\hline W5-O50 & $1.884(14)$ & W15-O11 & $1.89(2)$ \\
\hline W6-O30 & $1.961(14)$ & W15-O12 & $1.818(17)$ \\
\hline W6-O44 & $1.877(15)$ & W15-O13 & $2.381(14)$ \\
\hline
\end{tabular}




\begin{tabular}{|c|c|c|c|}
\hline W6-O45 & $1.701(16)$ & W16-O3 & $1.737(16)$ \\
\hline W6-O46 & $1.904(14)$ & W16-O4 & $1.882(16)$ \\
\hline W6-O47 & $2.358(14)$ & W16-O5 & $1.934(14)$ \\
\hline W6-O55 & $1.870(14)$ & W16-O9 & $1.863(15)$ \\
\hline W7-O31 & $2.019(14)$ & W16-O10 & $1.880(16)$ \\
\hline W7-O42 & $1.966(14)$ & W16-O13 & $2.369(14)$ \\
\hline W7-O43 & $1.743(15)$ & Co1-O1W & $2.146(18)$ \\
\hline W7-O44 & $1.918(15)$ & $\mathrm{Co} 1-\mathrm{O} 2 \mathrm{~W}$ & $2.086(18)$ \\
\hline W7-O48 & $2.348(13)$ & Co1-O3W & $2.108(18)$ \\
\hline W7-O54 & $1.813(14)$ & Co1-O22 & $2.085(17)$ \\
\hline W8-O8 & $1.968(15)$ & Co1-N1 & $2.14(2)$ \\
\hline W8-O19 & $1.866(15)$ & Co1-N3 & $2.13(2)$ \\
\hline W8-O20 & $1.705(17)$ & P1-O47 & $1.536(15)$ \\
\hline W8-O21 & $1.905(15)$ & $\mathrm{P} 1-\mathrm{O} 48$ & $1.540(15)$ \\
\hline W8-O28 & $2.370(15)$ & P1-O49 & $1.554(15)$ \\
\hline W8-O32 & $1.868(14)$ & $\mathrm{P} 1-\mathrm{O} 56$ & $1.614(14)$ \\
\hline W9-O7 & $1.971(17)$ & $\mathrm{P} 2-\mathrm{O} 13$ & $1.584(15)$ \\
\hline W9-O17 & $1.930(15)$ & $\mathrm{P} 2-\mathrm{O} 26$ & $1.512(14)$ \\
\hline W9-O18 & $1.714(16)$ & $\mathrm{P} 2-\mathrm{O} 27$ & $1.534(15)$ \\
\hline W9-O19 & $1.911(15)$ & $\mathrm{P} 2-\mathrm{O} 28$ & $1.536(15)$ \\
\hline O50-W1-O52 ${ }^{\mathrm{i}}$ & $164.8(7)$ & O18-W9-O26 & $172.0(6)$ \\
\hline O50-W1-O53 ${ }^{\mathrm{i}}$ & $89.3(6)$ & O18-W9-O33 & $98.9(7)$ \\
\hline O50-W1-O55 & $93.9(6)$ & O19-W9-O7 & $84.3(7)$ \\
\hline O50-W1-O56 & $85.6(6)$ & O19-W9-O17 & $156.8(7)$ \\
\hline $\mathrm{O} 52^{\mathrm{i}}-\mathrm{W} 1-\mathrm{O} 53^{\mathrm{i}}$ & $83.9(6)$ & O19-W9-O26 & $84.9(6)$ \\
\hline $\mathrm{O} 52^{\mathrm{i}}-\mathrm{W} 1-\mathrm{O} 56$ & $79.8(5)$ & O33-W9-O7 & $162.0(7)$ \\
\hline O53 ${ }^{\mathrm{i}}-\mathrm{W} 1-\mathrm{O} 56$ & $79.8(6)$ & O33-W9-O17 & $91.2(7)$ \\
\hline O55-W1-O52i & $89.1(6)$ & O33-W9-O19 & $88.9(6)$ \\
\hline O55-W1-O53 ${ }^{\mathrm{i}}$ & $164.0(7)$ & O33-W9-O26 & $82.0(6)$ \\
\hline O55-W1-O56 & $84.8(6)$ & O12-W10-O26 & $79.0(5)$ \\
\hline O57-W1-O50 & $94.8(7)$ & O15-W10-O12 & $82.4(7)$ \\
\hline O57-W1-O52 ${ }^{\mathrm{i}}$ & $99.9(7)$ & O15-W10-O17 & $158.7(6)$ \\
\hline O57-W1-O53 ${ }^{\mathrm{i}}$ & $101.0(7)$ & O15-W10-O26 & $85.8(6)$ \\
\hline O57-W1-O55 & $94.4(7)$ & O16-W10-O12 & $96.3(7)$ \\
\hline O57-W1-O56 & $179.1(6)$ & O16-W10-O15 & $102.1(7)$ \\
\hline O51-W1A-O52 & $97.4(6)$ & O16-W10-O17 & $97.3(7)$ \\
\hline O51-W1A-O53 ${ }^{\mathrm{i}}$ & $91.0(6)$ & O16-W10-O26 & $170.3(7)$ \\
\hline O51-W1A-O54 ${ }^{\mathrm{i}}$ & $91.9(6)$ & O16-W10-O34 & $101.9(7)$ \\
\hline O51-W1A-O56 & $91.8(6)$ & O17-W10-O12 & $87.0(7)$ \\
\hline O51-W1A-O56 ${ }^{\mathrm{i}}$ & $176.7(6)$ & O17-W10-O26 & $74.0(6)$ \\
\hline O52-W1A-O56 & $90.2(5)$ & O34-W10-O12 & $161.8(6)$ \\
\hline O53 ${ }^{\mathrm{i}}-\mathrm{W} 1 \mathrm{~A}-\mathrm{O} 52$ & $167.9(6)$ & O34-W10-O15 & $92.3(7)$ \\
\hline$O 53^{\mathrm{i}}-\mathrm{W} 1 \mathrm{~A}-\mathrm{O} 56^{\mathrm{i}}$ & $90.3(6)$ & O34-W10-O17 & $92.1(8)$ \\
\hline
\end{tabular}




\begin{tabular}{|c|c|c|c|}
\hline O53 ${ }^{\mathrm{i}}-\mathrm{W} 1 \mathrm{~A}-\mathrm{O} 56$ & $81.0(5)$ & O34-W10-O26 & $83.2(6)$ \\
\hline O54 ${ }^{\mathrm{i}}-\mathrm{W} 1 \mathrm{~A}-\mathrm{O} 52$ & $90.2(6)$ & O11-W11-O27 & $81.8(6)$ \\
\hline $\mathrm{O} 54^{\mathrm{i}}-\mathrm{W} 1 \mathrm{~A}-\mathrm{O} 53^{\mathrm{i}}$ & $98.1(6)$ & O14-W11-O11 & $98.3(7)$ \\
\hline O54 ${ }^{\mathrm{i}}-\mathrm{W} 1 \mathrm{~A}-\mathrm{O} 56^{\mathrm{i}}$ & $90.8(6)$ & O14-W11-O15 & $101.6(7)$ \\
\hline O54 $4^{\mathrm{i}}-\mathrm{W} 1 \mathrm{~A}-\mathrm{O} 56$ & $176.2(6)$ & O14-W11-O25 & $100.3(7)$ \\
\hline O56 ${ }^{\mathrm{i}}-\mathrm{W} 1 \mathrm{~A}-\mathrm{O} 52$ & $80.8(6)$ & O14-W11-O27 & $173.8(6)$ \\
\hline O56 ${ }^{\mathrm{i}}-\mathrm{W} 1 \mathrm{~A}-\mathrm{O} 56$ & $85.4(5)$ & O14-W11-O29 & $98.3(7)$ \\
\hline O32-W2-O48 & $83.7(6)$ & O15-W11-O11 & $84.8(7)$ \\
\hline O40-W2-O32 & $85.7(6)$ & O15-W11-O25 & $157.8(6)$ \\
\hline O40-W2-O48 & $84.3(6)$ & O15-W11-O27 & $84.6(6)$ \\
\hline O40-W2-O53 & $89.0(6)$ & O25-W11-O11 & $88.7(7)$ \\
\hline $\mathrm{O} 41-\mathrm{W} 2-\mathrm{O} 32$ & $96.5(7)$ & O25-W11-O27 & $73.5(6)$ \\
\hline $\mathrm{O} 41-\mathrm{W} 2-\mathrm{O} 40$ & $102.4(7)$ & O29-W11-O11 & $163.1(7)$ \\
\hline O41-W2-O42 & $99.6(7)$ & O29-W11-O15 & $88.9(7)$ \\
\hline O41-W2-O48 & $173.3(6)$ & O29-W11-O25 & $91.2(7)$ \\
\hline O41-W2-O53 & $98.4(7)$ & O29-W11-O27 & $82.0(6)$ \\
\hline O42-W2-O32 & $86.8(7)$ & O10-W12-O27 & $80.7(6)$ \\
\hline O42-W2-O40 & $157.4(6)$ & O23-W12-O10 & $83.6(7)$ \\
\hline O42-W2-O48 & $73.7(5)$ & O23-W12-O27 & $82.0(6)$ \\
\hline O42-W2-O53 & $92.8(6)$ & O24-W12-O10 & $95.9(7)$ \\
\hline O53-W2-O32 & $164.9(6)$ & O24-W12-O23 & $101.7(7)$ \\
\hline O53-W2-O48 & $81.8(5)$ & O24-W12-O25 & $101.0(7)$ \\
\hline O33-W3-O49 & $84.2(6)$ & O24-W12-O27 & $174.7(6)$ \\
\hline O38-W3-O33 & $88.7(7)$ & O24-W12-O30 & $101.3(7)$ \\
\hline O38-W3-O40 & $156.9(6)$ & $\mathrm{O} 25-\mathrm{W} 12-\mathrm{O} 10$ & $88.9(7)$ \\
\hline O38-W3-O49 & $71.9(6)$ & $\mathrm{O} 25-\mathrm{W} 12-\mathrm{O} 23$ & $156.7(6)$ \\
\hline O38-W3-O52 & $91.2(6)$ & $\mathrm{O} 25-\mathrm{W} 12-\mathrm{O} 27$ & $75.0(6)$ \\
\hline O39-W3-O33 & $96.9(7)$ & O30-W12-O10 & $162.4(6)$ \\
\hline O39-W3-O38 & $101.0(7)$ & O30-W12-O23 & $89.3(7)$ \\
\hline O39-W3-O40 & $101.8(7)$ & O30-W12-O25 & $91.4(7)$ \\
\hline O39-W3-O49 & $172.8(6)$ & O30-W12-O27 & $82.5(6)$ \\
\hline O39-W3-O52 & $97.2(7)$ & O9-W13-O28 & $80.5(5)$ \\
\hline O40-W3-O33 & $85.4(6)$ & O21-W13-O9 & $87.0(7)$ \\
\hline O40-W3-O49 & $85.3(5)$ & O21-W13-O28 & $73.5(6)$ \\
\hline O40-W3-O52 & $89.2(6)$ & O22-W13-O9 & $94.6(7)$ \\
\hline O52-W3-O33 & $165.7(6)$ & O22-W13-O21 & $100.1(7)$ \\
\hline O52-W3-O49 & $82.1(6)$ & O22-W13-O23 & $101.9(7)$ \\
\hline O34-W4-O49 & $81.5(6)$ & O22-W13-O28 & $172.0(7)$ \\
\hline O36-W4-O34 & $85.3(7)$ & O22-W13-O31 & $101.2(7)$ \\
\hline O36-W4-O38 & $155.3(6)$ & O23-W13-O9 & $83.6(6)$ \\
\hline O36-W4-O49 & $84.2(6)$ & O23-W13-O21 & $156.7(6)$ \\
\hline O37-W4-O34 & $96.1(7)$ & O23-W13-O28 & $83.9(6)$ \\
\hline O37-W4-O36 & $102.1(7)$ & O31-W13-O9 & $164.1(6)$ \\
\hline
\end{tabular}




\begin{tabular}{|c|c|c|c|}
\hline O37-W4-O38 & $101.3(7)$ & O31-W13-O21 & $92.0(7)$ \\
\hline O37-W4-O49 & $173.0(7)$ & O31-W13-O23 & $91.2(6)$ \\
\hline O37-W4-O51 & $100.1(7)$ & O31-W13-O28 & $84.1(5)$ \\
\hline O38-W4-O34 & $84.8(7)$ & O2-W14-O4 & $103.1(7)$ \\
\hline O38-W4-O49 & $72.0(5)$ & O2-W14-O6 & $101.7(8)$ \\
\hline O51-W4-O34 & $163.5(6)$ & O2-W14-O7 & $102.1(8)$ \\
\hline O51-W4-O36 & $94.2(7)$ & O2-W14-O8 & $102.9(8)$ \\
\hline O51-W4-O38 & $89.0(7)$ & O2-W14-O13 & $171.2(6)$ \\
\hline O51-W4-O49 & $82.0(6)$ & O4-W14-O13 & $71.1(6)$ \\
\hline O29-W5-O46 & $86.1(7)$ & O6-W14-O4 & $91.2(7)$ \\
\hline O29-W5-O47 & $83.6(6)$ & O6-W14-O13 & $72.3(6)$ \\
\hline O35-W5-O29 & $97.6(7)$ & O7-W14-O4 & $154.8(7)$ \\
\hline O35-W5-O36 & $100.3(7)$ & O7-W14-O6 & $85.5(8)$ \\
\hline O35-W5-O46 & $101.9(7)$ & O7-W14-O13 & $84.1(6)$ \\
\hline O35-W5-O47 & $175.6(6)$ & O8-W14-O4 & $86.2(7)$ \\
\hline O35-W5-O50 & $98.9(7)$ & O8-W14-O6 & $155.3(7)$ \\
\hline O36-W5-O29 & $86.1(7)$ & O8-W14-O7 & $86.5(7)$ \\
\hline O36-W5-O46 & $157.3(6)$ & O8-W14-O13 & $83.6(6)$ \\
\hline O36-W5-O47 & $84.0(6)$ & O1-W15-O5 & $101.2(8)$ \\
\hline O46-W5-O47 & $74.0(5)$ & O1-W15-O6 & $101.4(8)$ \\
\hline O50-W5-O29 & $163.5(7)$ & O1-W15-O11 & $101.6(8)$ \\
\hline O50-W5-O36 & $92.2(7)$ & O1-W15-O12 & $103.4(8)$ \\
\hline O50-W5-O46 & $89.3(6)$ & O1-W15-O13 & $170.0(7)$ \\
\hline O50-W5-O47 & $79.9(6)$ & O5-W15-O6 & $88.4(8)$ \\
\hline O30-W6-O47 & $82.3(5)$ & O5-W15-O13 & $71.9(5)$ \\
\hline O44-W6-O30 & $87.2(6)$ & O6-W15-O13 & $71.6(6)$ \\
\hline O44-W6-O46 & $158.3(6)$ & O11-W15-O5 & $87.6(7)$ \\
\hline O44-W6-O47 & $84.8(6)$ & O11-W15-O6 & $157.0(7)$ \\
\hline O45-W6-O30 & $98.4(6)$ & O11-W15-O13 & $85.6(6)$ \\
\hline O45-W6-O44 & $100.8(7)$ & O12-W15-O5 & $155.3(6)$ \\
\hline O45-W6-O46 & $100.3(7)$ & O12-W15-O6 & $85.6(8)$ \\
\hline O45-W6-O47 & $174.4(6)$ & O12-W15-O11 & $88.7(7)$ \\
\hline O45-W6-O55 & $99.0(7)$ & O12-W15-O13 & $83.5(6)$ \\
\hline O46-W6-O30 & $84.7(6)$ & O3-W16-O4 & $100.0(7)$ \\
\hline O46-W6-O47 & $74.2(5)$ & O3-W16-O5 & $100.4(7)$ \\
\hline O55-W6-O30 & $162.4(6)$ & O3-W16-O9 & $103.7(7)$ \\
\hline O55-W6-O44 & $92.3(6)$ & O3-W16-O10 & $103.6(7)$ \\
\hline O55-W6-O46 & $89.4(6)$ & O3-W16-O13 & $168.8(6)$ \\
\hline O55-W6-O47 & $80.2(6)$ & O4-W16-O5 & $88.0(7)$ \\
\hline O31-W7-O48 & $81.1(5)$ & O4-W16-O13 & $72.1(6)$ \\
\hline O42-W7-O31 & $82.7(6)$ & O5-W16-O13 & $71.9(6)$ \\
\hline O42-W7-O48 & $71.9(5)$ & O9-W16-O4 & $86.1(7)$ \\
\hline O43-W7-O31 & $95.7(6)$ & O9-W16-O5 & $155.9(6)$ \\
\hline
\end{tabular}




\begin{tabular}{|l|l|l|l|}
\hline O43-W7-O42 & $101.5(6)$ & O9-W16-O10 & $88.2(6)$ \\
\hline O43-W7-O44 & $100.6(7)$ & O9-W16-O13 & $84.0(6)$ \\
\hline O43-W7-O48 & $172.9(6)$ & O10-W16-O4 & $156.3(7)$ \\
\hline O43-W7-O54 & $100.0(7)$ & O10-W16-O5 & $87.9(7)$ \\
\hline O44-W7-O31 & $87.2(6)$ & O10-W16-O13 & $84.5(6)$ \\
\hline O44-W7-O42 & $156.5(6)$ & O2W-Co1-O1W & $91.3(8)$ \\
\hline O44-W7-O48 & $85.6(6)$ & O2W-Co1-O3W & $93.0(8)$ \\
\hline O54-W7-O31 & $164.0(6)$ & O2W-Co1-N1 & $90.8(9)$ \\
\hline O54-W7-O42 & $91.3(6)$ & O2W-Co1-N3 & $87.7(8)$ \\
\hline O54-W7-O44 & $92.7(6)$ & O3W-Co1-O1W & $174.5(7)$ \\
\hline O54-W7-O48 & $82.9(6)$ & O3W-Co1-N1 & $86.9(8)$ \\
\hline O8-W8-O28 & $80.4(6)$ & O3W-Co1-N3 & $93.3(8)$ \\
\hline O19-W8-O8 & $84.7(6)$ & O22-Co1-O1W & $86.6(6)$ \\
\hline O19-W8-O21 & $157.2(7)$ & O22-Co1-O2W & $176.4(8)$ \\
\hline O19-W8-O28 & $84.8(6)$ & O22-Co1-O3W & $89.2(6)$ \\
\hline O19-W8-O32 & $89.2(6)$ & O22-Co1-N1 & $92.2(8)$ \\
\hline O20-W8-O8 & $97.0(7)$ & O22-Co1-N3 & $89.3(8)$ \\
\hline O20-W8-O19 & $102.7(7)$ & N1-Co1-O1W & $89.6(8)$ \\
\hline O20-W8-O21 & $99.7(7)$ & N3-Co1-O1W & $90.2(8)$ \\
\hline O20-W8-O28 & $171.9(6)$ & N3-Co1-N1 & $178.5(9)$ \\
\hline O20-W8-O32 & $101.2(7)$ & O47-P1-O48 & $111.4(8)$ \\
\hline O21-W8-O8 & $88.5(7)$ & O47-P1-O49 & $110.8(8)$ \\
\hline O21-W8-O28 & $72.6(6)$ & O47-P1-O56 & $106.5(8)$ \\
\hline O32-W8-O8 & $161.6(7)$ & O48-P1-O49 & $111.8(8)$ \\
\hline O32-W8-O21 & $90.5(7)$ & O48-P1-O56 & $108.3(8)$ \\
\hline O32-W8-O28 & $81.8(6)$ & O49-P1-O56 & $107.8(8)$ \\
\hline O7-W9-O26 & $80.8(6)$ & O26-P2-O13 & $106.3(8)$ \\
\hline O17-W9-O7 & $88.7(7)$ & O26-P2-O27 & $112.6(8)$ \\
\hline O17-W9-O26 & $72.2(6)$ & O26-P2-O28 & $112.2(8)$ \\
\hline O18-W9-O7 & $98.9(7)$ & O27-P2-O13 & $108.2(8)$ \\
\hline O18-W9-O17 & $99.8(7)$ & O27-P2-O28 & $111.7(8)$ \\
\hline O18-W9-O19 & $103.1(7)$ & O28-P2-O13 & $105.4(8)$ \\
\hline
\end{tabular}


Figure S11. The solid-state circular dichroism (CD) spectra of 1. Each sample for CD spectrum was collected from all crystals of a single batch, totally four samples.

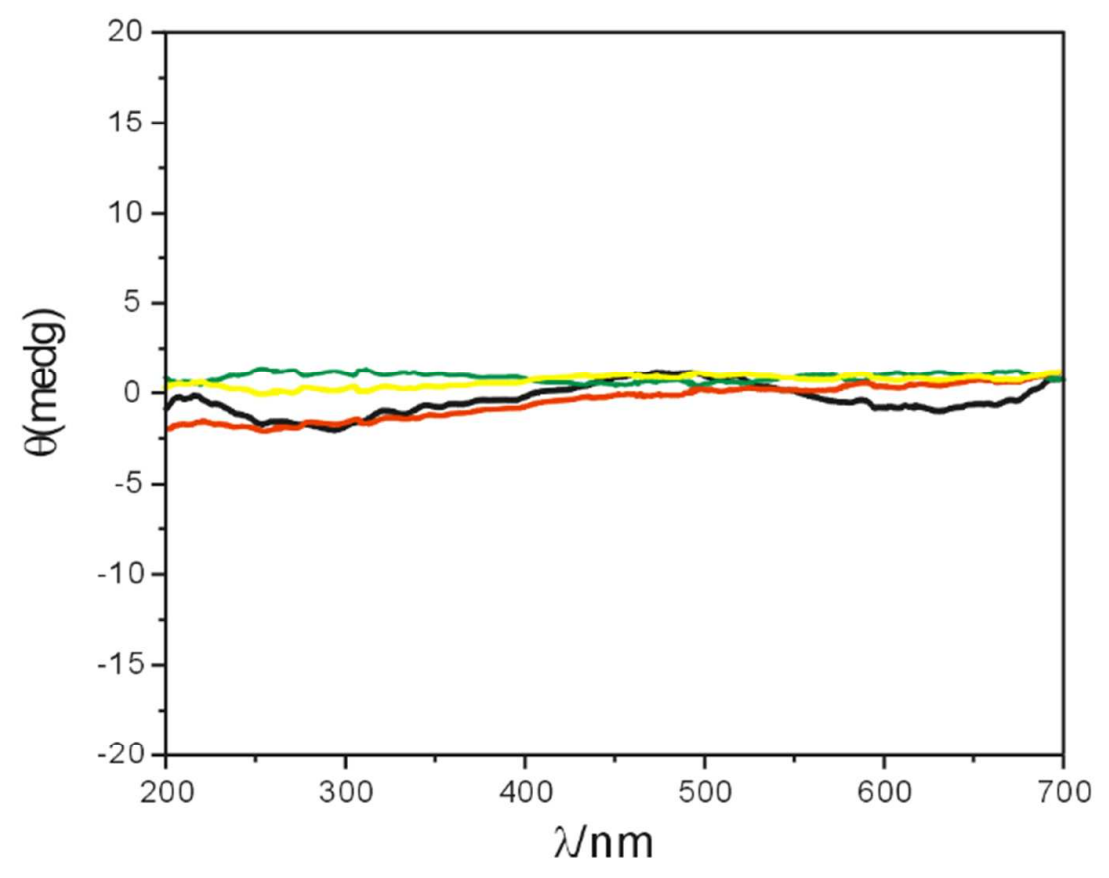


Figure S12. The microscopy photo of 1.

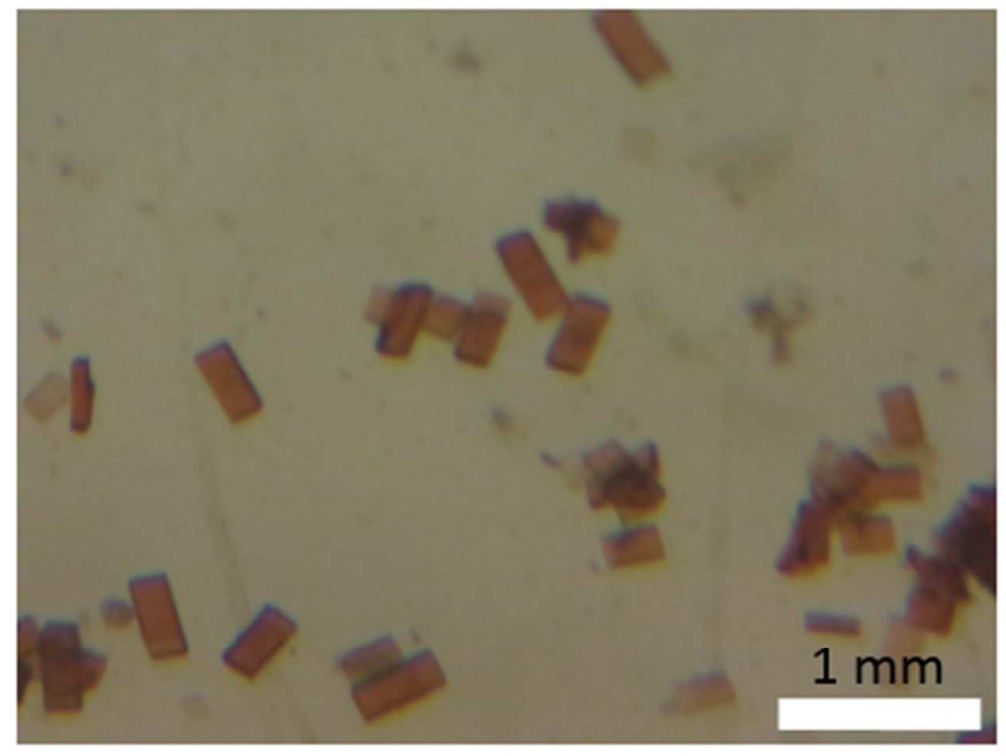

